\title{
Investigation of Particle-in-Cell Acceleration Techniques for Plasma Simulations
}

\author{
David D. Marshall* \\ California Polytechnic State University, San Luis Obispo, CA 93407-0352, USA \\ Douglas B. VanGilder ${ }^{\dagger}$ \\ Combustion Research and Flow Technology, Inc., Pipersville, PA 18947, USA
}

\begin{abstract}
COLISEUM is an application framework that integrates plasma propagation schemes and arbitrary 3D surface geometries. Using Particle-in-Cell (PIC) schemes to model the plasma propagation high fidelity modeling of the plasma and its interactions with the surfaces is possible. In order to improve the computational performance of the Particle-in-Cell scheme with Direct Simulation Monte Carlo collision modeling (PIC-DSMC) within COLISEUM, AQUILA, acceleration techniques have been developed that significantly decrease the amount of CPU time needed to obtain a steady-state solution. These techniques have been demonstrated to decrease the CPU time from 3 to 24 times with little appreciable differences in the global particle properties and number densities. This work investigates the differences in the local plasma properties that result from the application of the different acceleration techniques. Results show that the subcycling acceleration scheme does accurately capture the macroscopic flow properties (such as particle counts and species number densities) and the velocity distributions in the lower density regions of the flow field. However, the higher density regions of the flow field (such as in the main beam of the plasma source) show significant differences that are believed to be associated with the simplifying assumptions used in the original collision modeling scheme within the PIC-DSMC module AQUILA.
\end{abstract}

\section{Nomenclature}

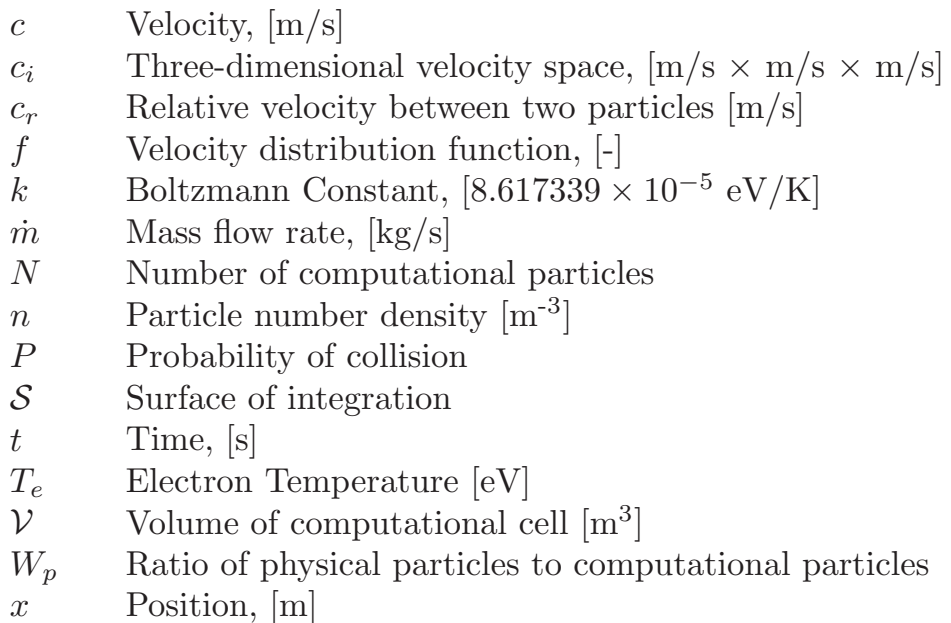

\footnotetext{
*Assistant Professor, Aerospace Engineering Department, California Polytechnic State University, San Luis Obispo, CA 93407-0352, Member AIAA.

${ }^{\dagger}$ Research Scientist, Combustion Research and Flow Technology, Inc., 6210 Keller's Church Road, Pipersville, PA 18947, Member AIAA.
} 


\begin{tabular}{ll}
\multicolumn{2}{l}{ Subscripts } \\
max & Maximum value \\
$s$ & Surface property \\
Conventions & \\
DSMC & Direct Simulation Monte Carlo Collision Modeling \\
MCC & Monte Carlo Collision Modeling \\
NTC & No-Time-Counter Collision Model \\
PIC & Particle-in-Cell Modeling Technique for Particles \\
SCN & COLISEUM cases run without subcyling \\
SCY & COLISEUM cases run with subcyling \\
VDF & Velocity Distribution Function \\
Symbols & \\
$\epsilon_{0}$ & Permittivity of free space, $\approx 8.8542 \times 10^{-12} \mathrm{~F} / \mathrm{m}$ \\
$\phi$ & Electrostatic potential $[\mathrm{V}]$ \\
$\rho_{e}$ & Electron charge density $\left[\mathrm{C} / \mathrm{m}^{3}\right]$ \\
$\rho_{i}$ & Ion charge density $\left[\mathrm{C} / \mathrm{m}^{3}\right]$ \\
$\sigma_{T}$ & Total collision cross-section $\left[\mathrm{m}^{2}\right]$ \\
$\tau$ & Characteristic time $[\mathrm{s}]$
\end{tabular}

\section{Introduction}

7 HE Air Force Research Laboratory has developed an application framework that integrates plasma propagation schemes with arbitrary $3 \mathrm{D}$ surface geometries ${ }^{1}$ in order to investigate the interactions between the plasma plume from electric propulsion thrusters and the spacecraft. A hybrid Particle-in-Cell plasma model within COLISEUM, called AQUILA, ${ }^{2}$ is the basis of this work. The COLISEUM framework is used to model the thrusters in space environment for prediction of the interactions and also to model the thrusters in vacuum chambers in order to validate the models used in COLISEUM against experimental data. In order to improve the computational performance of the AQUILA model, acceleration techniques have been developed that significantly decrease the amount of CPU time needed to get the simulation to a steady-state. These schemes have been demonstrated to decrease the CPU time by up to 24 times with little appreciable differences in the global particle properties.

Two previous studies have been performed on the acceleration schemes within COLISEUM. Gibbons et al. ${ }^{3}$ demonstrated the subcycling technique in which the slower moving neutrals are propagated at a larger time step than the faster moving ions. Gibbons et al. ${ }^{4}$ demonstrated the use of the subcycling scheme with a scheme that decoupled the modeling of surface interactions from the plume propagation. It utilizes particle sources from the surfaces in place of the self-consistent surface interaction modeling. Both of these schemes are intended to provide increased convergence rates to a steady-state solution. The desire is to end up with the same final plasma distribution with or without the acceleration techniques. The present study intends to provide a detailed analysis of the resulting plasma properties and any differences in the local plasma properties that result from the application of the subcycling acceleration techniques.

\section{Computational Techniques}

This section provides a brief overview of the major computational techniques within COLISEUM and AQUILA obtained from the User's Manuals.

\section{A. COLISEUM Framework}

COLISEUM is a computational framework that provides a tool that can be used to model the interaction of plasmas with arbitrary surfaces in three-dimensional space. ${ }^{3}$ These simulations can be of a plasma in a contained domain or in an open domain. This allows the simulation of experiments in vacuum chambers as well as plasmas in the low density space environment. The primary focus of COLISEUM is to investigate the erosion associated with the plasma particles impacting surfaces, known as sputtering, as well as the 
re-deposition of this material on other surfaces. COLISEUM provides the integration of the CAD surface modeling, the plasma propagation, and the sputtering of material within one consistent framework.

\section{Surface Modeling}

The surface model can be input in a variety of standard formats including ANSYS and ABAQUS formats. In addition, surface properties are also specified in order to differentiate the various materials that may compose each surface. In order to accurately model the sputtering, additional material information must be specified that describes the interaction between particles that may be impacting surfaces and the material that the surfaces are composed of. This is known as the material interaction parameters in COLISEUM.

The sputtering models in COLISEUM are based on standard models from Roussel et al. ${ }^{5}$ and Gardner et al., ${ }^{6}$ Kannenberg et al., ${ }^{7}$ and Yamamura et al. ${ }^{8}$ Coupling the sputtering models with the re-deposition process has been included in order to account for how the re-deposited material may itself be sputtered. This allows a more accurate model of the final surface deposition characteristics. Fife et al. ${ }^{1}$ have also developed an iterative scheme to model resputtering of the deposited material.

\section{Plasma Modeling}

The plasma modeling within COLISEUM has two major components to it. The first is the modeling of the source. Sources are surfaces within the geometry from which particles will be emitted. To model sources, a velocity distribution function, VDF, must be specified throughout the surface of the source. In general, the VDF is a function of space, time, and velocity, $f(\vec{x}, \vec{c}, t)$. This is used to determine the mass flow rate, $\dot{m}$, as

$$
\dot{m}_{s}=\int_{\mathcal{S}} \int_{c_{i}} f_{s}(\vec{x}, \vec{c}, t) d c_{i} d \mathcal{S}
$$

where $c_{i}$ is the three-dimensional velocity space, and $\mathcal{S}$ is the surface of the source. The VDF does not need a specified direction since COLISEUM uses the surface normals from the geometry. COLISEUM provides surface models for common sources: (1) user specified flux information typically from experimental data, (2) user specified flux and velocity information typically from experimental data, and (3) a shifted Maxwellian distribution with the velocity shift normal to the surface.

The second major component of the plasma modeling within COLISEUM is the plasma simulation itself. COLISEUM was developed with the idea of supporting any number of plasma modeling schemes. One of the original models is the prescribed plume model which simply imports previously obtained plasma properties of the flow field. The particle fluxes to the surfaces are calculated by mapping the solution to the surface geometry. The other original model is a ray tracing model which traces the particle trajectory from the sources without accounting for the electrostatic potential field forces or particle collisions. The particle fluxes to the surfaces are then determined.

As COLISEUM has matured, more sophisticated plasma modeling modules have been developed. DRACO from Virginia Tech $^{9}$ is a Cartesian cell based, finite-element Particle-in-Cell Monte Carlo Collision (PIC$\mathrm{MCC}$ ) simulation. AQUILA from $\mathrm{MIT}^{10}$ is an unstructured tetrahedral cell based, finite-element PIC-DSMC simulation. It is AQUILA that is being used as the basis of this investigation.

\section{B. Particle Propagation Scheme}

To propagate the particles, two separate schemes are used. The neutrals and ions are treated as particles and are propagated via a particle tracking technique. The electrons are treated as a fluid. The electrons are described by the Boltzmann relation

$$
n_{e}=n_{e, 0} \exp \left(\frac{e \phi}{k T_{e}}\right)
$$

The time integration scheme used within AQUILA to propagate the plasma particles is the standard leap frog scheme $^{11}$ which is second order accurate in time. The electrostatic forces are modeled using the electrostatic potential equation with the inclusion of space-charge effects ${ }^{10}$

$$
\nabla^{2} \phi=\frac{\rho_{e}-\rho_{i}}{\epsilon_{0}}
$$


where $\phi$ is the electrostatic potential, $\rho_{e}$ is the electron charge density, $\rho_{i}$ is the ion charge density, and $\epsilon_{0}$ is the permittivity of free space. A finite element formulation is used to solve this potential equation with a Newton-method type scheme to handle the nonlinear nature of the resulting equations.

\section{Collision Modeling}

The collision modeling within AQUILA ${ }^{12}$ is based on the No-Time-Counter, NTC, method of Bird. ${ }^{13}$ The probability of a collision between two particles is given as

$$
P=\frac{W_{p}\left(\sigma_{T} c_{r}\right) \Delta t}{\mathcal{V}}
$$

where $W_{p}$ is the ratio of physical particles to computational particles, $\sigma_{T}$ is the total collision cross-section, $c_{r}$ is the relative speed between the two particles, and $\mathcal{V}$ is the volume of the computational cell containing particles. Similarly, the maximum probability of a collision is

$$
P_{\max }=\frac{W_{p}\left(\sigma_{T} c_{r}\right)_{\max } \Delta t}{\mathcal{V}}
$$

The NTC scheme samples only a fraction of the total number of particle pairs in the computational cell, and adjusts the probability of collision of the sampled particle pairs accordingly. Within COLISEUM, only $P_{\max } N_{p} N_{q}$ particle pairs are chosen from species $p$ and $q$. Thus, the lower the maximum probability of collision, the fewer collision samples are taken. The resulting collision probability for a sampled collision pair is then

$$
P=\frac{\sigma_{t} c_{r}}{\left(\sigma_{T} c_{r}\right)_{\max }}
$$

Notice that this scheme will sample the appropriate number of collision pairs only when an accurate maximum probability has been determined. Before such time, too few collisions pairs will be sampled resulting in the lower probability events (but not insignificant events) being under represented. Therefore, this scheme will produce accurate collision rates only after a sufficient number of pairs have been sampled so that the maximum probability term has been reasonably determined. Once this occurs the collision calculations should then reasonably capture all of the desired collision events.

Within AQUILA, each particle species (neutrals and ions of the same atom are considered to be different species in addition to different atoms) can have its own physical to computational particle weighting. This means that $W_{p}$ from above corresponds to the larger of the two weightings in the collision pair. Also, while the lower weighted pair, $W_{q}$ in the collision will always be altered by the collision, when one occurs, the probability of the higher weighted particle being altered by the collsion is

$$
P_{W_{p}}=\frac{W_{q}}{W_{p}}
$$

This results in the possibility of momentum not being conserved for individual collisions, but momentum will be conserved on average with a sufficient number of collisions.

Finally, for all collision related calculations, a simple accept/reject scheme can be used on the probabilities.

\section{Subcycling Scheme}

The subcycling scheme within COLISEUM utilizes the fact that there is a significant difference between the collision times scales and the ion characteristic time. The ion characteristic time is based on the spatial resolution of the computational grid which is being used to model the electrostatic potential field. In order to keep the electrostatic forces on the particles varying smoothly as they travel through the domain, the ions should not travel more than a third of a cell within one time step. For the simulation to be modeled in this paper, the ion velocity is $20 \mathrm{~km} / \mathrm{s}$ and the neutral velocity is $200 \mathrm{~m} / \mathrm{s}$. For a characteristic length of the smallest volume of $0.01 \mathrm{~m}$, this results in the ion characteristic time of $5 \times 10^{-7} \mathrm{~s}$ and the neutral characteristic time of $5 \times 10^{-5} \mathrm{~s}$. This is a factor of 100 difference between the two. 
Using the following relations for the elastic neutral-neutral and neutral-ion collisions ${ }^{14}$ to characterize the collision cross-sections

$$
\begin{aligned}
\sigma_{X e-X e}^{\mathrm{el}} & =\frac{2.117 \times 10^{-18}}{c_{r}^{0.24}} \\
\sigma_{X e-X e^{+}}^{\mathrm{el}} & =\frac{8.2807 \times 10^{-16}}{c_{r}}
\end{aligned}
$$

and for the charge-exchange collisions between neutrals and ions ${ }^{15}$

$$
\sigma_{X e-X e^{+}}^{\mathrm{cex}}=1.1872 \times 10^{-20}\left[-23.3 \log \left(c_{r}\right)+188.81\right]
$$

a mean time to collision can be determined as

$$
\tau=\frac{1}{n \sigma c_{r}}
$$

Table 1 shows the characteristic times for the simulation to be modeled in this paper and a maximum particle number density of $10^{18} \mathrm{~m}^{-3}$. Included in these calculations is the high speed neutrals that will result from previous charge exchange collisions. Their number density will be shown to be 100 times less than the maximum particle number density. Thus, the neutrals in the collision calculations could have the low speed

\begin{tabular}{|c|c|c|c|}
\hline Collision Type & $c_{r}[\mathrm{~m} / \mathrm{s}]$ & $\sigma\left[\mathrm{m}^{2}\right]$ & $\tau[\mathrm{s}]$ \\
\hline Xe-Xe Elastic & 200 & $5.94 \times 10^{-19}$ & $8.42 \times 10^{-3}$ \\
\hline Xe-Xe Elastic & 20000 & $1.97 \times 10^{-19}$ & $2.56 \times 10^{-2}$ \\
\hline $\mathrm{Xe} \mathrm{Xe}^{+}$Elastic & 10000 & $8.20 \times 10^{-20}$ & $1.21 \times 10^{-1}$ \\
\hline $\mathrm{Xe} \mathrm{Xe}^{+}$Elastic & 20000 & $4.18 \times 10^{-20}$ & $1.21 \times 10^{-3}$ \\
\hline $\mathrm{Xe}-\mathrm{Xe}^{+}$Charge Exchange & 10000 & $1.14 \times 10^{-18}$ & $8.81 \times 10^{-3}$ \\
\hline $\mathrm{Xe}-\mathrm{Xe}^{+}$Charge Exchange & 20000 & $1.05 \times 10^{-18}$ & $4.75 \times 10^{-5}$ \\
\hline
\end{tabular}
$200 \mathrm{~m} / \mathrm{s}$ value or the high speed $20000 \mathrm{~m} / \mathrm{s}$ value. Clearly a simulation time step less than $4.7 \times 10^{-5} \mathrm{~s}$ is needed to resolve the collision time scales.

Table 1. Collision Characteristic Times

Therefore, there is only one physical phenomena that requires a time step in the order of $10^{-7} \mathrm{~s}$, and that is capturing the electrostatic forces applied to the ions.

Computing one complete computational cycle encompasses the following steps

1. Subcycle Fast Particles
(a) Move Fast Particles
(b) Inject Fast Particles
(c) Update Electrostatic Fields

2. Move Slow Particles

3. Inject Slow Particles

\section{Perform Collisions}

By only moving the slow particles a fraction of the number times that the fast particles must be moved as well as performing the collisions on the coarse time step a significant amount of computational effort is saved. 


\section{E. Velocity Distribution Function Probe}

In order to determine the local plasma properties, a new probe was introduced into the AQUILA probe architecture. This probe samples a region in space (currently a computational cell) and stores the velocity of every particle of a specified type that resides within the cell. The frequency of sampling can be adjusted as well as the start and end times of the sampling. Once the sampling is completed, the probe sorts the particles into bins of user specified sizes. The results can be written out as a table of the non-empty bins, or as a Tecplot formatted structured grid data file. Further processing is possible with this data if only binning on particle speed is desired.

\section{Results}

The following results are all for the same test problem. First, solution convergence is demonstrated by using a fine time step that is on the order of the characteristic time step of the electrostatic forces. It is also demonstrated that further refinement of the time step does not result in any appreciable change in the solution. Second, a solution is presented with a coarse time step that is on the order of the collision time scale. This solution is compared to the fine time step solution. Next, a solution is presented using the subcycling scheme discussed above with the ions moving at the fine time step and the neutrals moving at the coarse time step.

\section{A. Test Problem Description}

The test problem is a highly simplified geometry based on a plasma source within a vacuum chamber. Figure 1 shows the surface meshes associated with the test problem. The plasma source is a small cylinder with the cylinder axis aligned with the z-axis. The plasma is emitted in the positive z-direction from that particular face of the cylinder. The vacuum chamber is simplified to a cylinder with the cylinder axis again aligned with the z-axis. The plasma source is firing towards one end of the chamber, while the opposite end of the chamber is a particle sink such that any particle that hits that surface leaves the computational domain.

The chamber has a diameter of $1.5 \mathrm{~m}$ and a length of $2 \mathrm{~m}$. The plasma source has a diameter of $0.1 \mathrm{~m}$ and a length of $0.1 \mathrm{~m}$. The distance between the plasma source face and the chamber face is $1.3 \mathrm{~m}$

The plasma source is composed of two particle types, low speed neutrals and high speed ions. Both are modeled using the drifting Maxwellian source model within COLISEUM. ${ }^{16}$ The neutral drift ve-

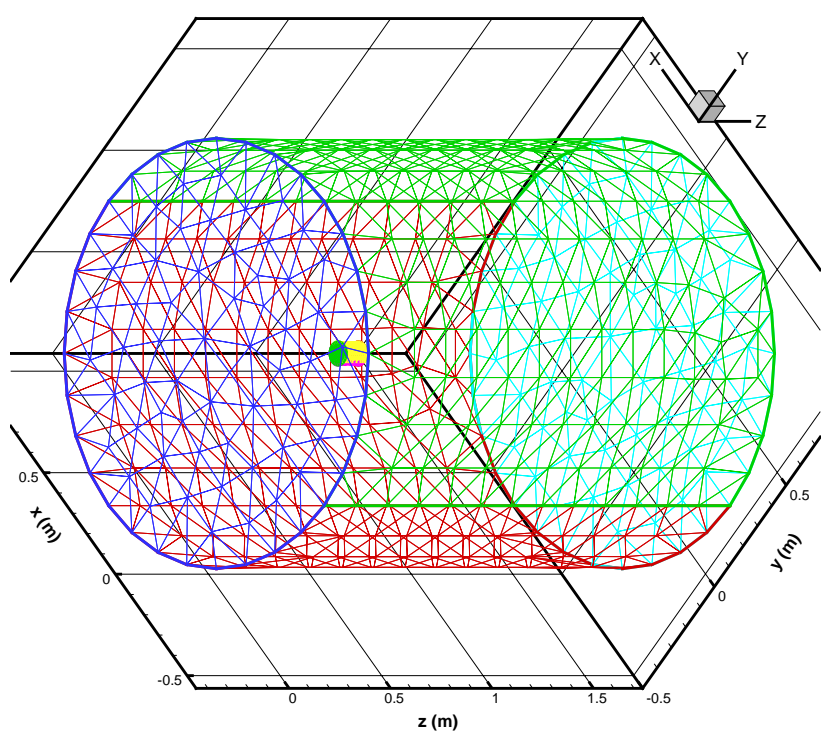

Figure 1. Simple Chamber Geometry locity is $200 \mathrm{~m} / \mathrm{s}$ and temperature is $700 \mathrm{~K}$. The ion drift velocity is $20 \mathrm{~km} / \mathrm{s}$ and temperature is $10 \mathrm{eV}$. The ratio of particle weights between the neutrals and ions is $300: 1$ and the physical to computational particle ratio for the neutrals is $6.00 \times 10^{11}$. Finally the ion mass flow rate is $5.0 \times 10^{-7} \mathrm{~kg} / \mathrm{s}$, and the neutral mass flow rate is $1.0 \times 10^{-7} \mathrm{~kg} / \mathrm{s}$.

The electrostatic potential is modeled using the quasi-neutral model within AQUILA ${ }^{12}$ instead of the non-equilibrium model mentioned previously. This applies the quasi-neutrality assumption and inverts Boltzmann's equation to obtain an expression for the electrostatic potential

$$
\phi=\phi_{0}+\frac{k T_{e}}{e} \ln \left(\frac{n_{e}}{n_{e 0}}\right)
$$

where the electron temperature, $T_{e}$, is set to $2 \mathrm{eV}$. The reference electron number density and potential is specified to be at a potential of $0 \mathrm{~V}$ just in front of the thruster face. 
In order to examine the similarities of the local properties of the plasma between the three cases, the velocity distribution for the neutrals and ions were obtained $0.1 \mathrm{~m}$ in front of the plasma source as well as $0.28 \mathrm{~m}$ above the thruster face. The first sampling will examine the plume modeling capabilities, while the second sampling will examine the capabilities to model the plasma outside of the main plasma beam.

The computer that performed these simulations for the timing results is a dual processor AMD Opteron 242 system with 2 GB of RAM with an additional 2 GB of swap space. Use of the machine was minimized while the cases were running, and all cases resided in physical memory, so the swap space was not utilized except to move other non-essential applications out of RAM at the start of the simulation.

In order to quickly distinguish between the various cases to be run, the acronym SCN will designate cases without the use of subcycling and SCY will designate the cases with subcycling.

\section{B. Solution Convergence Demonstration}

With the baseline fine time step for this simulation established as $2.5 \times 10^{-7} \mathrm{~s}$, three cases were run to demonstrate the convergence of the solution at this time step. One case was at twice the baseline time step, $5.0 \times 10^{-7} \mathrm{~s}$, and one at half the baseline time step, $1.25 \times 10^{-7} \mathrm{~s}$. Each case was computed to a final computational time of $0.25 \mathrm{~s}$. Table 2 shows the collision rates for the three cases. There is very little difference between all of the collision rates for the three cases. The differences between the cases is most certainly due to the statistical scatter associated with these types of schemes. Notice that halving or doubling the step size approximately doubled or halved the amount of time required to calculate the solution.

Table 2. Collision Rates for Solution Convergence Cases

\begin{tabular}{|c|c|c|c|c|c|c|}
\hline Sch & $\begin{array}{l}\text { Compute } \\
\text { Time [hr] }\end{array}$ & $\begin{array}{c}\text { Time } \\
\text { Step }[\mathrm{s}]\end{array}$ & $\begin{array}{c}\text { Total } \\
\text { Collisions }[\# / \mathrm{s}]\end{array}$ & $\begin{array}{l}\mathrm{Xe}-\mathrm{Xe}^{+} \text {Charge } \\
\text { Exchange }[\# / \mathrm{s}]\end{array}$ & $\begin{array}{c}\mathrm{Xe}-\mathrm{Xe} \\
\text { Elastic }[\# / \mathrm{s}]\end{array}$ & $\begin{array}{c}\mathrm{Xe}-\mathrm{Xe}^{+} \\
\text {Elastic }[\# / \mathrm{s}]\end{array}$ \\
\hline $\mathrm{SCN}$ & 107.2 & $1.25 \times 10^{-7}$ & $5.301 \times 10^{7}$ & $5.046 \times 10^{7}$ & $2.519 \times 10^{5}$ & $2.293 \times 10^{6}$ \\
\hline $\mathrm{SCN}$ & 58.9 & $2.5 \times$ & $5.325 \times 10^{7}$ & $5.069 \times 10^{7}$ & $2.560 \times 10^{5}$ & $2.303 \times 10^{6}$ \\
\hline $\mathrm{SCN}$ & 30.9 & $5.0 \times 10^{-7}$ & $5.310 \times 10^{7}$ & $5.056 \times 10^{7}$ & $2.541 \times 10^{5}$ & $2.287 \times 10^{6}$ \\
\hline
\end{tabular}

Figure 2 shows the evolution of the total number of neutrals and ions as well as the total number of particles. These counts are nearly identical with any differences with the statistical scatter.

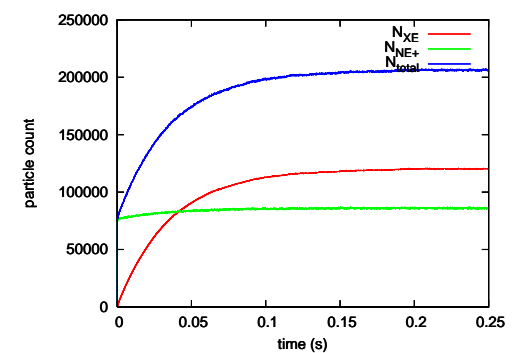

(a) $\mathrm{SCN} \Delta t=1.25 \times 10^{-7} \mathrm{~s}$

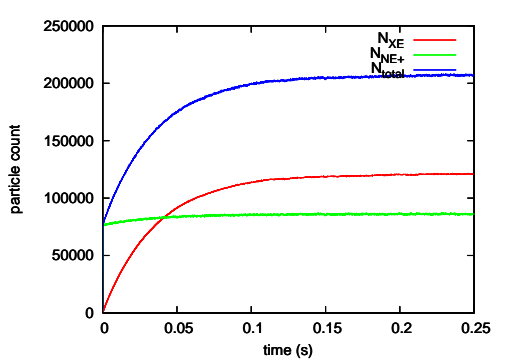

(b) $\operatorname{SCN} \Delta t=2.5 \times 10^{-7} \mathrm{~s}$

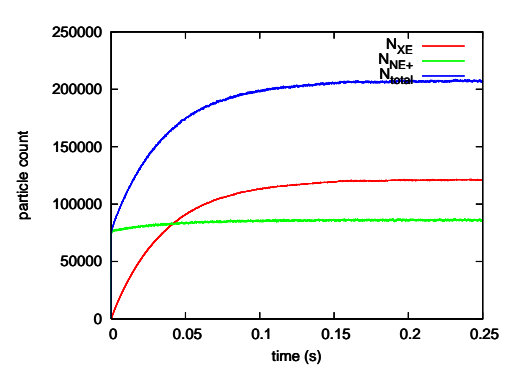

(c) $\mathrm{SCN} \Delta t=5.0 \times 10^{-7} \mathrm{~s}$

Figure 2. Global Particle Counts for Solution Convergence Cases

Figure 3 shows a contour plot of the final number density distribution for the neutrals for the three cases. The plane that is shown is the $\mathrm{x}-\mathrm{z}$ plane through center of the plasma source. All figures shown are for the same plane at the same location. Figure 4 shows a contour plot of the final number density distribution for the ions for the three cases, and Figure 5 shows a contour plot of the final electrostatic potential for the three cases. All of these figures also show only very minor differences between the three cases. The outer wings of the plume are captured in all three cases as can be seen in the ion number density and the electrostatic potential. In addition, the time step is clearly sufficient to accurately capture the ion trajectories. As can be seen, the ions are occupying the region behind the plasma source which can only occur if the time step is sufficiently small to allow the electrostatic forces to curve the trajectories of the charge-exchange ions away from the plasma source. The very low ion density immediately behind the ion source is due to the ions colliding with that surface and reflecting back as accommodated neutrals. 


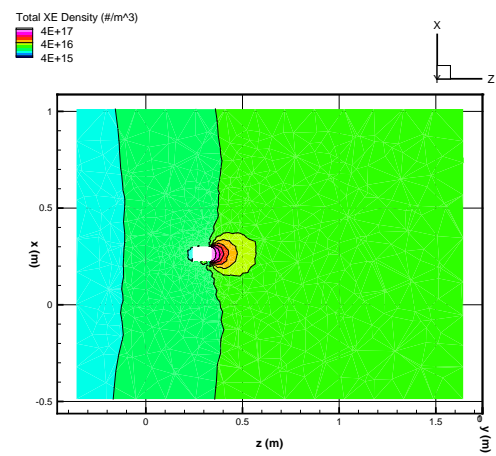

(a) $\operatorname{SCN} \Delta t=1.25 \times 10^{-7} \mathrm{~s}$

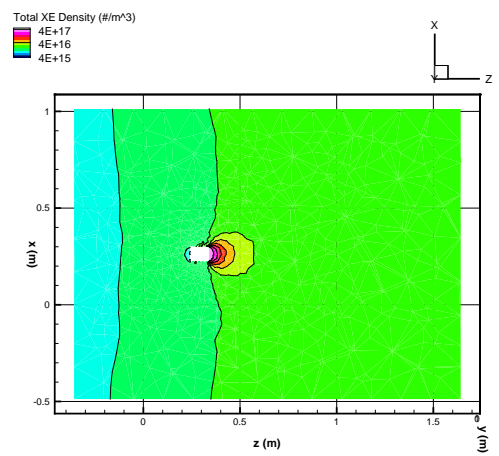

(b) $\operatorname{SCN} \Delta t=2.5 \times 10^{-7} \mathrm{~s}$

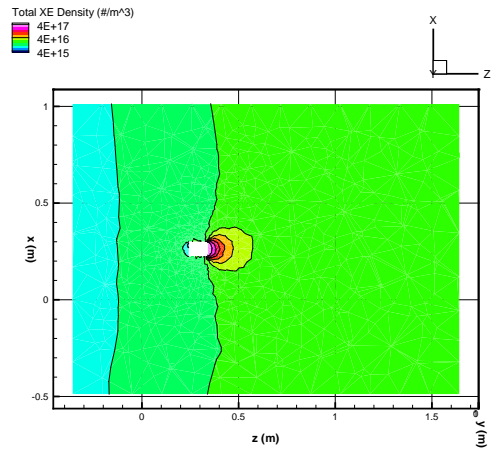

(c) $\mathrm{SCN} \Delta t=5.0 \times 10^{-7} \mathrm{~s}$

Figure 3. Final Neutral Number Density for Solution Convergence Cases

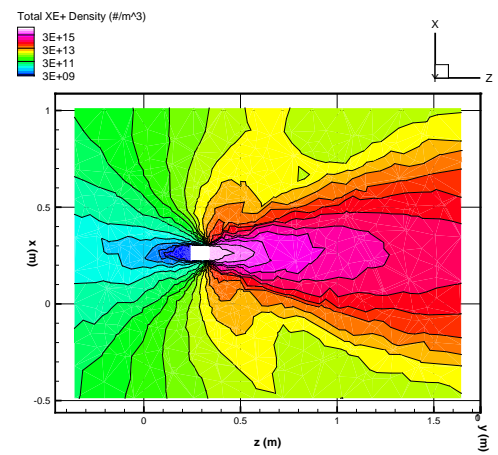

(a) $\operatorname{SCN} \Delta t=1.25 \times 10^{-7} \mathrm{~s}$

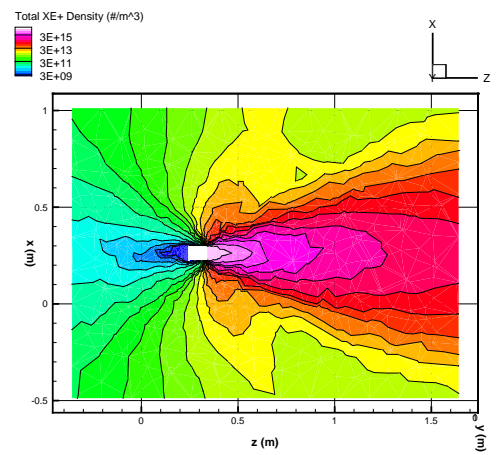

(b) $\mathrm{SCN} \Delta t=2.5 \times 10^{-7} \mathrm{~s}$

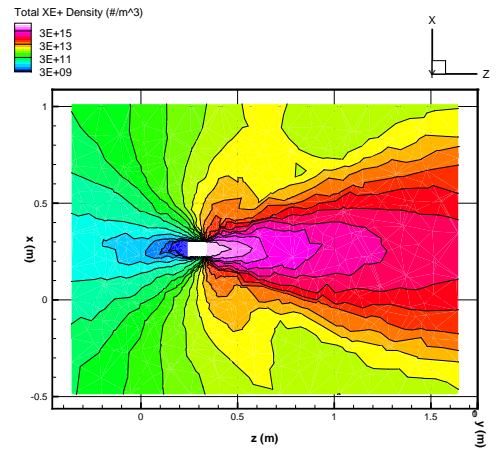

(c) $\mathrm{SCN} \Delta t=5.0 \times 10^{-7} \mathrm{~s}$

Figure 4. Final Ion Number Density for Solution Convergence Cases

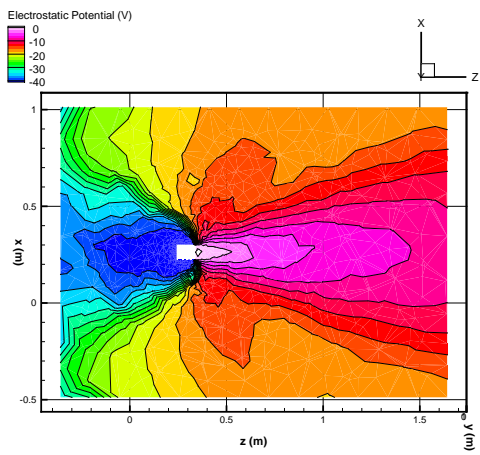

(a) $\mathrm{SCN} \Delta t=1.25 \times 10^{-7} \mathrm{~s}$

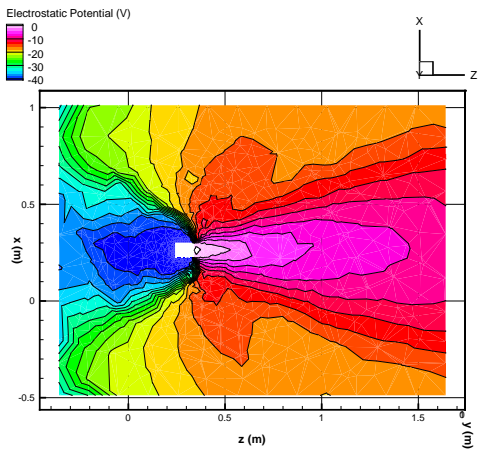

(b) $\mathrm{SCN} \Delta t=2.5 \times 10^{-7} \mathrm{~s}$

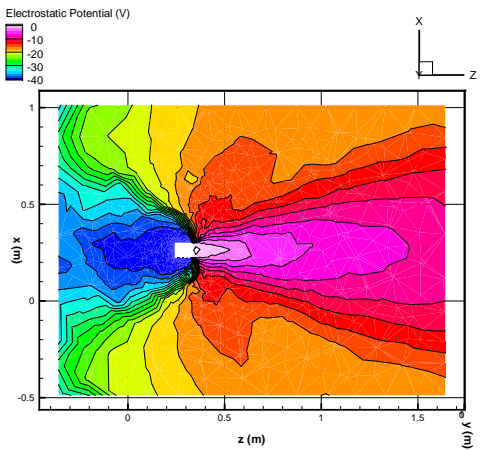

(c) $\mathrm{SCN} \Delta t=5.0 \times 10^{-7} \mathrm{~s}$

Figure 5. Final Electrostatic Potential for Solution Convergence Cases 
Next, the local properties of the plasma will be compared between the three cases. The sampling is performed using the VDF probe. Samples are obtained every $2.5 \times 10^{-5} \mathrm{~s}$, for this case every 100 time steps. Sampling is started after $0.4 \mathrm{~s}$ have elapsed, when the global particle count has reached a nearly steady state. The neutral sampling within the plume is shown in Figure 6 for both the velocity distribution as well as the speed distribution. Thehe velocity distribution is showing the outer edge of the velocity space domain that is populated with particles and the surfaces are colored by z-velocity since that is the primary velocity direction. There are a total of approximately 6100 particles being sampled in each case. Figure 7 shows the ion sampling within the plume, where a total of approximately 670,000 particles are being sampled in each case. Once again, there is very little differences between the three cases. A bimodal distribution is seen in the ion distribution with the lower speed ions being the result of the charge-exchange collisions between the neutrals and the ions. The corresponding high speed neutrals can be seen in the neutral distributions, but the clarity is obscured because of the relative low occurrence of these particles due to the computational particle weighting used for the neutrals.
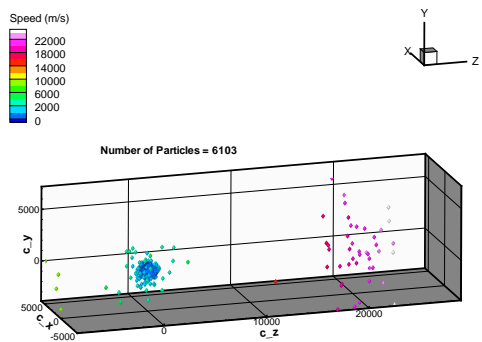
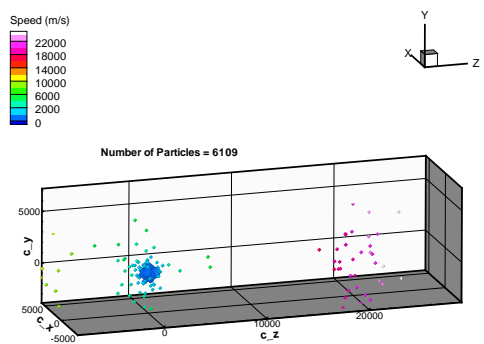

(b) SCN Velocity $\Delta t=2.5 \times 10^{-7} \mathrm{~s}$

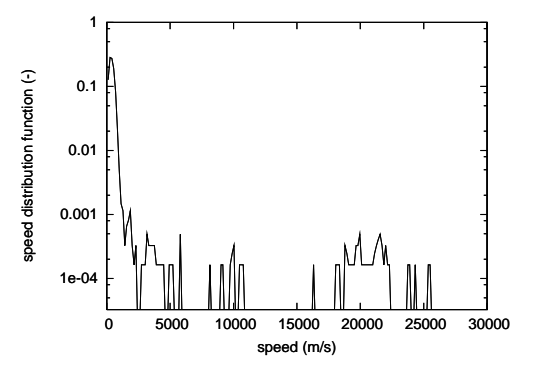

(e) SCN Speed $\Delta t=2.5 \times 10^{-7} \mathrm{~s}$

(e) SCN Sped $\Delta t=2.5 \times 10^{-7} \mathrm{~s}$
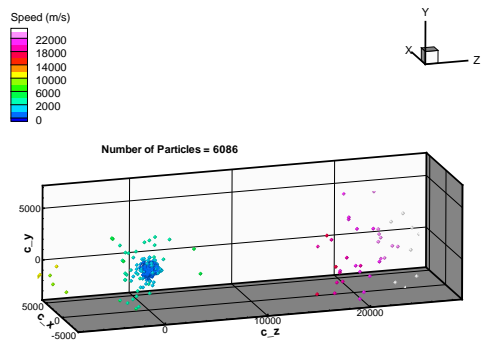

(a) SCN Velocity $\Delta t=1.25 \times 10^{-7} \mathrm{~s}$

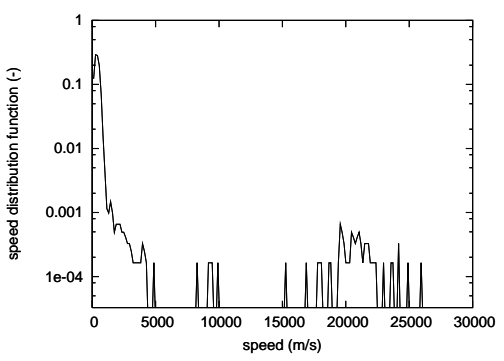

(d) SCN Speed $\Delta t=1.25 \times 10^{-7} \mathrm{~s}$ (c) SCN Velocity $\Delta t=5.0 \times 10^{-7} \mathrm{~s}$

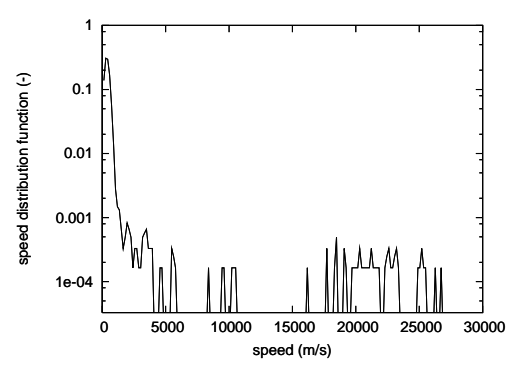

(f) SCN Speed $\Delta t=5.0 \times 10^{-7} \mathrm{~s}$

Figure 6. Neutral Velocity and Speed Distributions $0.1 \mathrm{~m}$ in Front of Thruster for Solution Convergence Cases

The neutral sampling outside the plume is shown in Figure 8 for both the velocity distribution as well as the speed distribution, with the same sampling configuration parameters used for the previous samplings. Figure 9 shows the ion sampling outside the plume. In this case there is very little difference between the three cases, but some minor differences do occur. The majority of the neutrals that are being sampled in this region are due to the ions accomodating and reflecting off of the walls. That is why the most probable velocity is so low. However, the $5.0 \times 10^{-7} \mathrm{~s}$ case does show some added noise in the higher velocities. Notice that at this time step, we are very close to the ion characteristic time step, so the increase in the time step size might have altered the ion trajectories enough to alter the neutral distribution in the higher speeds. There still exists a bimodal distribution in the ion velocity, but the number of low speed ions is significantly less than what were in front of the thruster, as expected. Also, the most probable speed of the ions has dropped from the $20 \mathrm{~km} / \mathrm{s}$ that it was in front of the thruster to around $4 \mathrm{~km} / \mathrm{s}$. This is because the ions that make it to this region of the flow are the low speed charge exchange ions that have been accelerated through the electrostatic potential field into this region. 

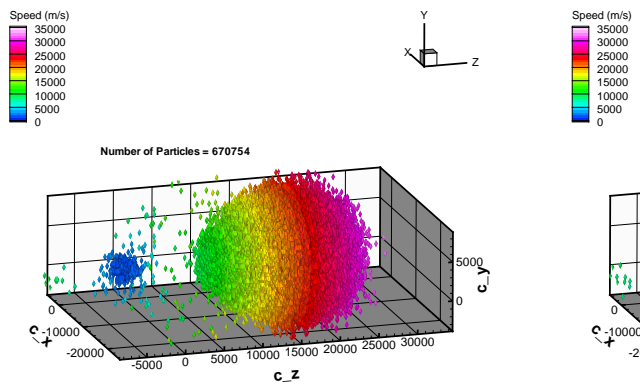

in
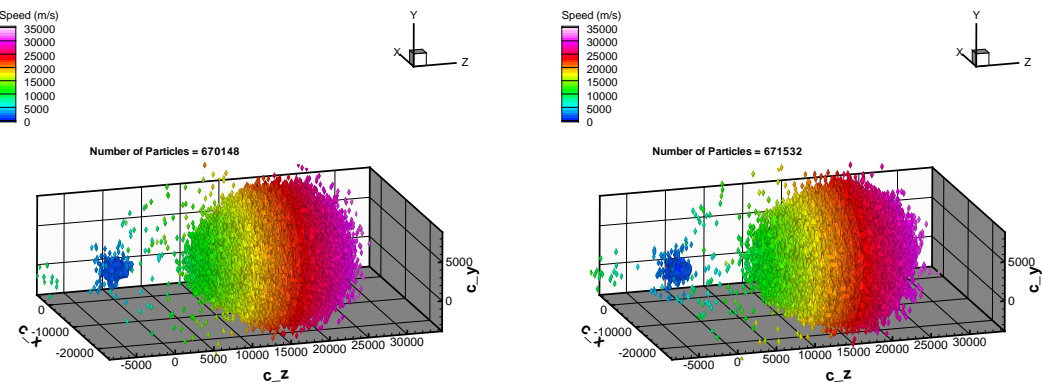

(a) SCN Velocity $\Delta t=1.25 \times 10^{-7} \mathrm{~s}$

(b) SCN Velocity $\Delta t=2.5 \times 10^{-7} \mathrm{~s}$

(c) SCN Velocity $\Delta t=5.0 \times 10^{-7} \mathrm{~s}$
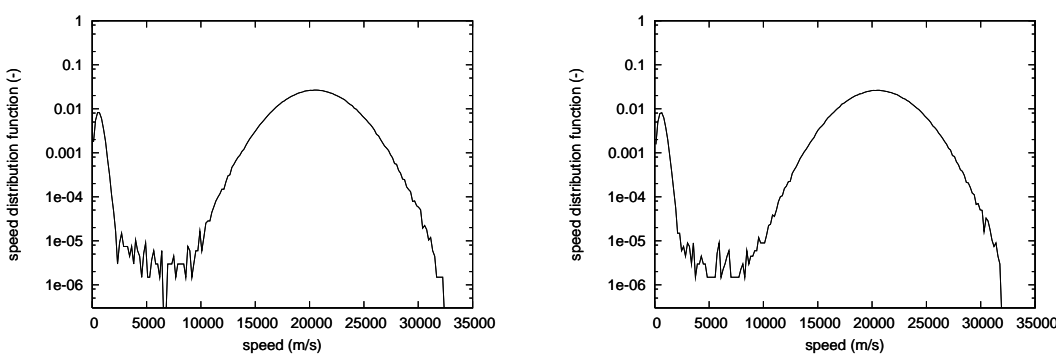

(d) SCN Speed $\Delta t=1.25 \times 10^{-7} \mathrm{~s}$

(e) SCN Speed $\Delta t=2.5 \times 10^{-7} \mathrm{~s}$

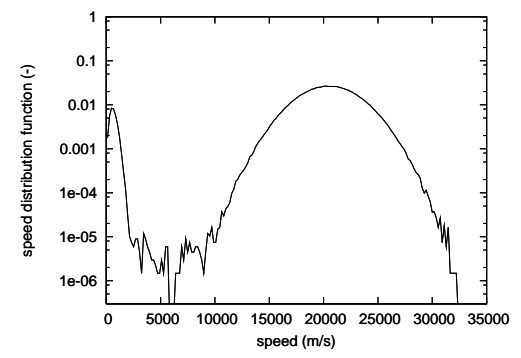

(f) SCN Speed $\Delta t=5.0 \times 10^{-7} \mathrm{~s}$

Figure 7. Ion Velocity and Speed Distributions $0.1 \mathrm{~m}$ in Front of Thruster for Solution Convergence Cases
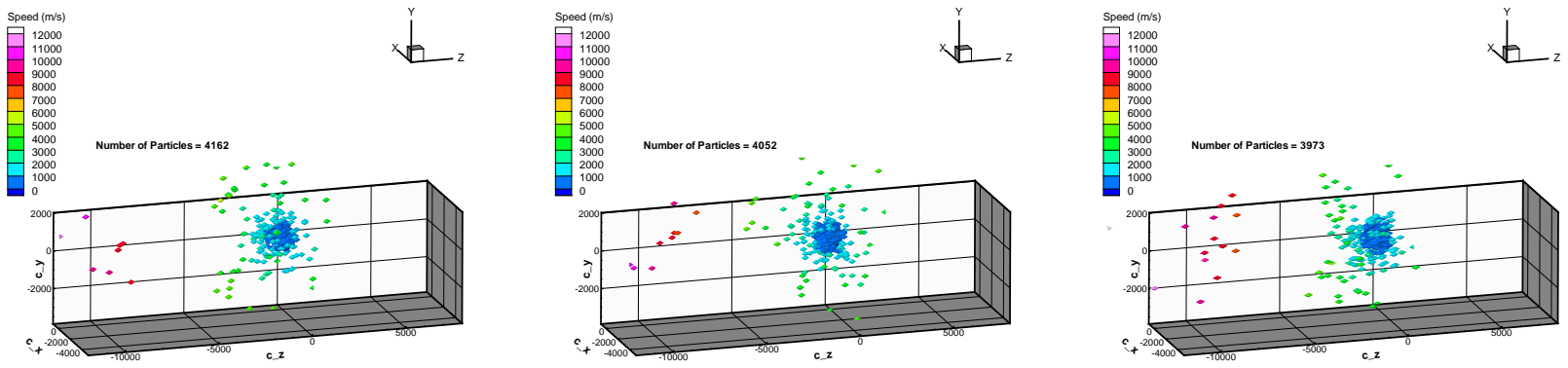

(a) SCN Velocity $\Delta t=1.25 \times 10^{-7} \mathrm{~s}$

(b) SCN Velocity $\Delta t=2.5 \times 10^{-7} \mathrm{~s}$

(c) SCN Velocity $\Delta t=5.0 \times 10^{-7} \mathrm{~s}$
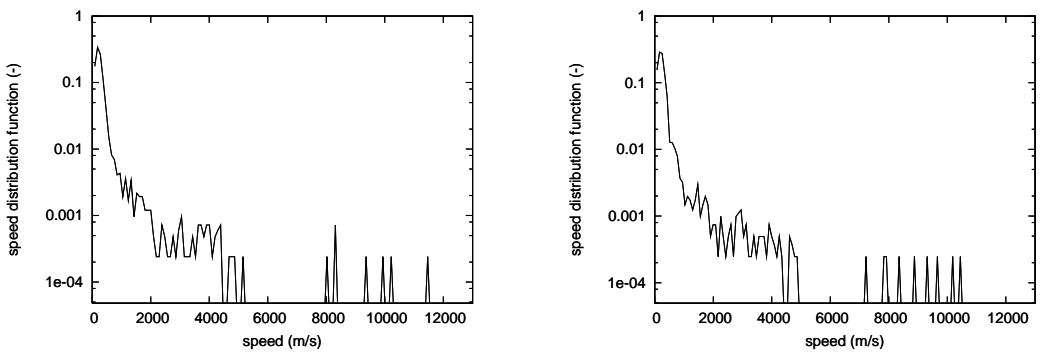

(d) SCN Speed $\Delta t=1.25 \times 10^{-7} \mathrm{~s}$

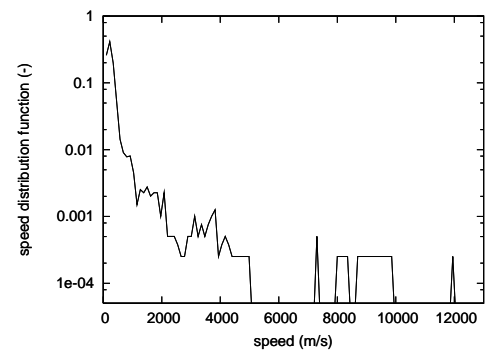

(f) $\mathrm{SCN}$ Speed $\Delta t=5.0 \times 10^{-7} \mathrm{~s}$

Figure 8. Neutral Velocity and Speed Distributions $0.28 \mathrm{~m}$ Above Thruster Face for Solution Convergence Cases 


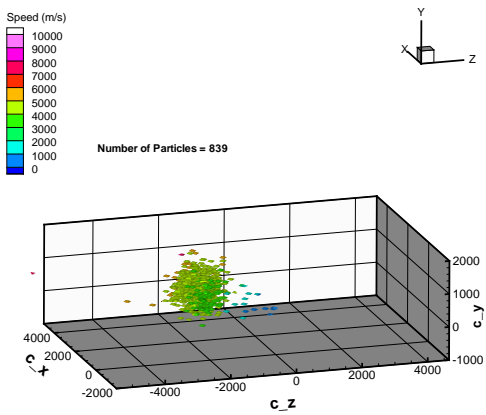

(a) SCN Velocity $\Delta t=1.25 \times 10^{-7} \mathrm{~s}$

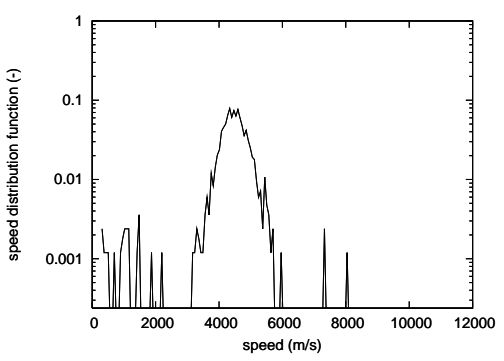

(d) SCN Speed $\Delta t=1.25 \times 10^{-7} \mathrm{~s}$

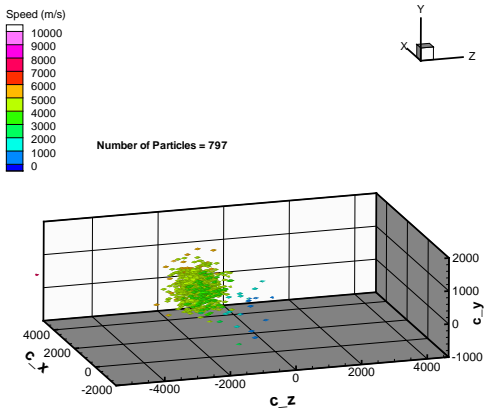

(b) SCN Velocity $\Delta t=2.5 \times 10^{-7} \mathrm{~s}$

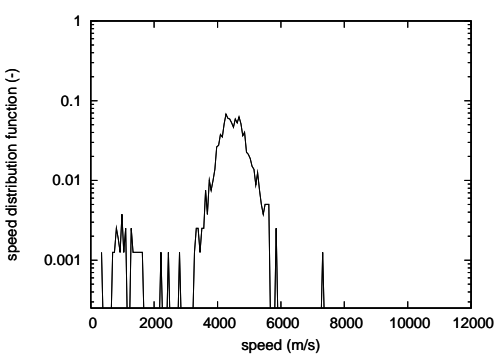

(e) SCN Speed $\Delta t=2.5 \times 10^{-7} \mathrm{~s}$

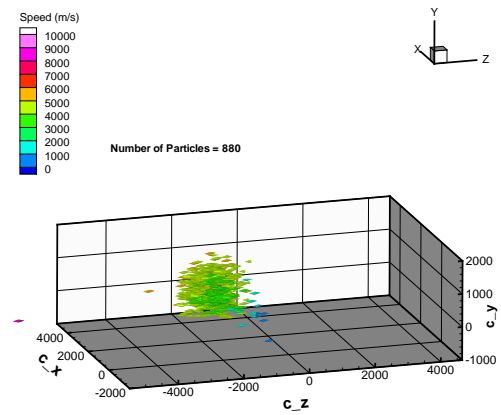

(c) SCN Velocity $\Delta t=5.0 \times 10^{-7} \mathrm{~s}$

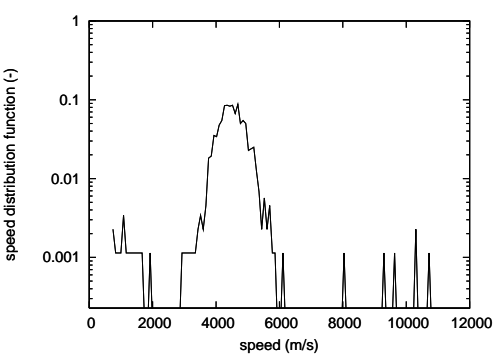

(f) $\mathrm{SCN}$ Speed $\Delta t=5.0 \times 10^{-7} \mathrm{~s}$

Figure 9. Ion Velocity and Speed Distributions $0.28 \mathrm{~m}$ Above Thruster Face for Solution Convergence Cases

\section{Coarse Time Step Solution}

Now the coarse time step case can be compared to the fine time step. It is understood that these results will have significant errors associated with the time step being too large to capture the correct ion trajectories. This case is still instructive in observing what features are not being captured by the coarse time step and for future discussions of features in the subcycling scheme that can be attributed to the coarse time step used for the neutral time scale.

For this case all particles and physical processes are propagated at a time step of $2.5 \times 10^{-5} \mathrm{~s}$. From the previous discussions, it is known that this time step is larger than the characteristic time associated with the electrostatic forces, $5.0 \times 10^{-7} \mathrm{~s}$, and is very close to the smallest characteristic time associated with the collision modeling, $4.7 \times 10^{-5}$ for most of the charge exchange collision events that occur. Table 3 shows the resulting collision rates along with the fine time step collision rates.

Table 3. Collision Rates for Coarse Time Step

\begin{tabular}{ccccccc} 
& $\begin{array}{c}\text { Compute } \\
\text { Scheme }\end{array}$ & $\begin{array}{c}\text { Time }[\mathrm{hr}] \\
\text { Timep [s] }\end{array}$ & $\begin{array}{c}\text { Total } \\
\text { Collisions }[\# / \mathrm{s}]\end{array}$ & $\begin{array}{c}\mathrm{Xe}-\mathrm{Xe}^{+} \text {Charge } \\
\text { Exchange [\#/s] }\end{array}$ & $\begin{array}{c}\mathrm{Xe}-\mathrm{Xe} \\
\text { Elastic [\#/s] }\end{array}$ & $\begin{array}{c}\mathrm{Xe}-\mathrm{Xe}^{+} \\
\text {Elastic [\#/s] }\end{array}$ \\
\hline SCN & 58.9 & $2.5 \times 10^{-7}$ & $5.325 \times 10^{7}$ & $5.069 \times 10^{7}$ & $2.560 \times 10^{5}$ & $2.303 \times 10^{6}$ \\
SCN & 1.73 & $2.5 \times 10^{-5}$ & $4.945 \times 10^{7}$ & $4.726 \times 10^{7}$ & $2.572 \times 10^{5}$ & $1.936 \times 10^{6}$
\end{tabular}

The first thing to notice from this table is that taking 100 times fewer time steps results in a significant decrease in compute time, by a factor of around 34. Unfortunately, only the Xe-Xe elastic collision rate is the same between the coarse and fine time steps. The elastic and charge exchange collisions associated with the $\mathrm{Xe}-\mathrm{Xe}^{+}$pairs differ between the two cases. Therefore by not adequately resolving the ion trajectory there has been a decrease in the collision rate associated with the ions. This could be caused by the ions traveling entirely through the high density region in front of the plasma source (where collisions are most likely) before the ions can participate in a significant number of collision events. Taking the nominal ion velocity of $20 \mathrm{~km} / \mathrm{s}$ and the time step of $2.5 \times 10^{-5} \mathrm{~s}$ yields a distance traveled by an ion of $0.5 \mathrm{~m}$. This is a significant distance from the plasma source and is certainly outside of the high density region found in the 
fine time step cases from Figures 3 and 4.

While significant differences are seen in the collision rates, Figure 10 shows that the evolution of the total number of neutrals and ions as well as the total number of particles is very similar. Thus, the differences between the two cases must be for only a small, but significant, fraction of the total particles.

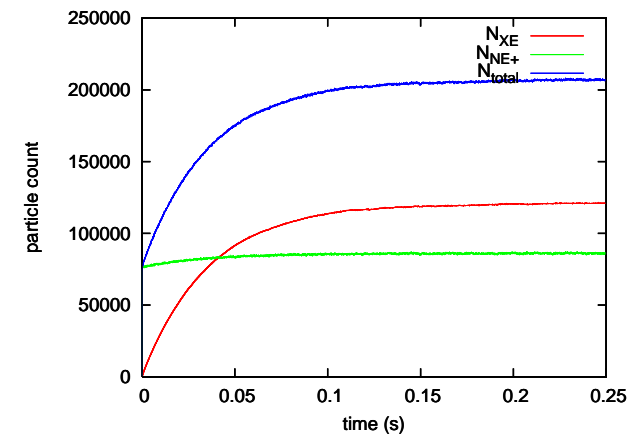

(a) $\operatorname{SCN} \Delta t=2.5 \times 10^{-7} \mathrm{~s}$

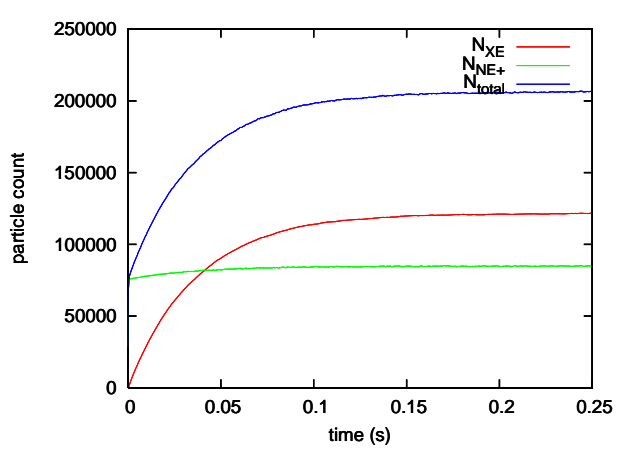

(b) $\mathrm{SCN} \Delta t=2.5 \times 10^{-5} \mathrm{~s}$

Figure 10. Global Particle Counts for Coarse Time Step

Figure 11 shows the final number density for the neutrals for the coarse and fine time step cases. Even for this case the neutral number density is fairly similar. It appears that the coarse time step does not have a significant effect on the overall neutral number density. This does not mean, however, that there is no effect. Since the collision rate is different, there is likely some difference in the velocity distribution function throughout the computational domain.

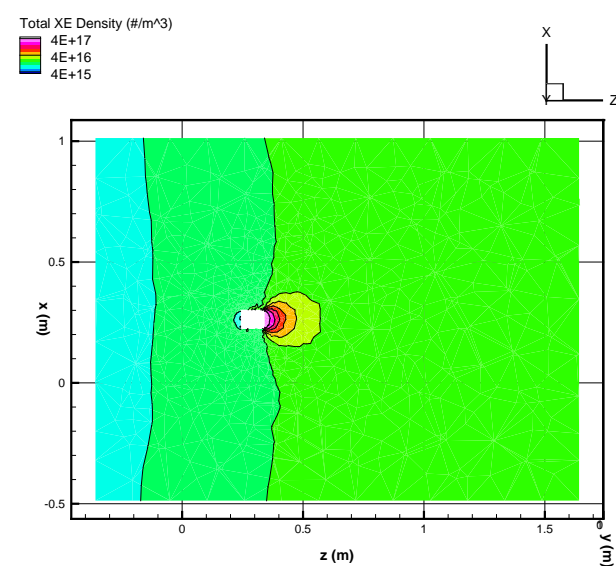

(a) $\mathrm{SCN} \Delta t=2.5 \times 10^{-7} \mathrm{~s}$

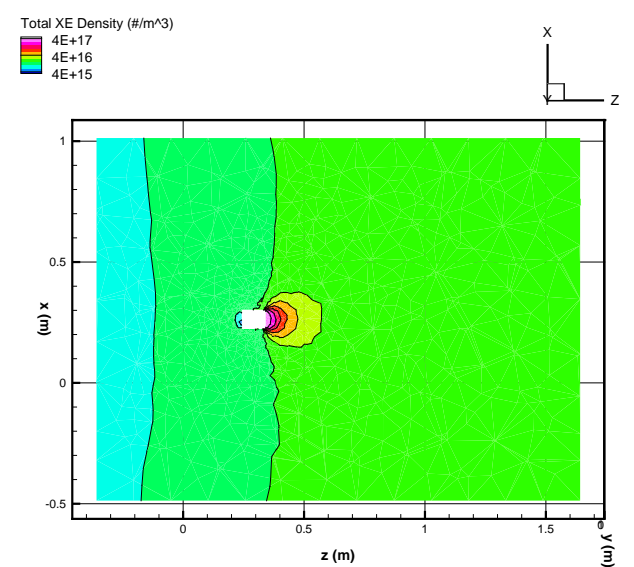

(b) SCN $\Delta t=2.5 \times 10^{-5} \mathrm{~s}$

Figure 11. Final Neutral Number Density for Coarse Time Step

Figure 12 shows the final number density for the ions for the coarse and fine time step cases. This shows a significant difference between the two cases. While the main beam region seems similar, the outer wings of the plume are certainly not captured as well in the coarse time step case. Also, with the time step so large, the ions cannot make the curved trajectory to collide with the back of the ion source, which results in the difference between the two cases in that region.

Figure 13 shows the final electrostatic potential for the coarse and fine time step cases. This again shows less accurate resolution of the outer wings of the plume region. Also an increased potential region behind the plasma source exists since the ions are not colliding with the back of the plasma source and becoming neutralized.

Next, the local properties of the plasma will be compared between the three cases. The neutral sampling within in the plume is shown in Figure 14 for both the velocity distribution as well as the speed distribution. The most significant difference here is that there is a significantly larger band of high velocity neutrals, around the $20-25 \mathrm{~km} / \mathrm{s}$ range, with a noticeable decrease in population in the $10 \mathrm{~km} / \mathrm{s}$ region for the coarse 


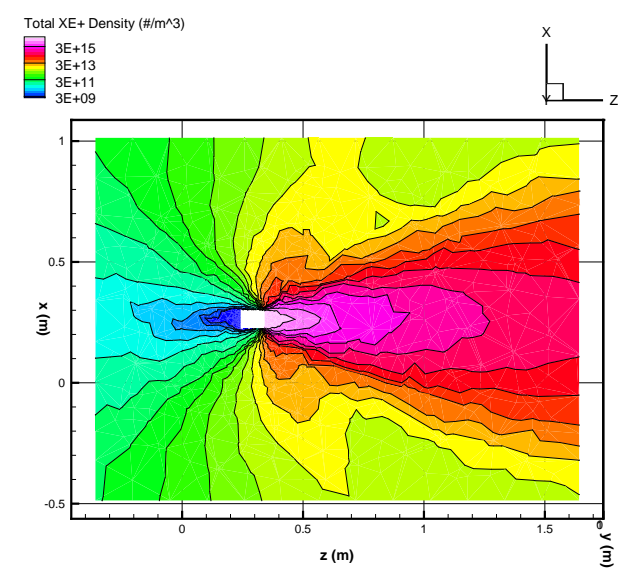

(a) $\mathrm{SCN} \Delta t=2.5 \times 10^{-7} \mathrm{~s}$

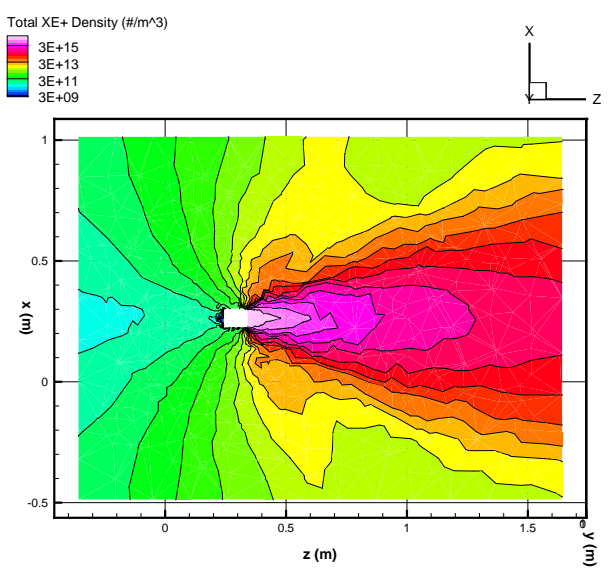

(b) $\operatorname{SCN} \Delta t=2.5 \times 10^{-5} \mathrm{~s}$

Figure 12. Final Ion Number Density for Coarse Time Step

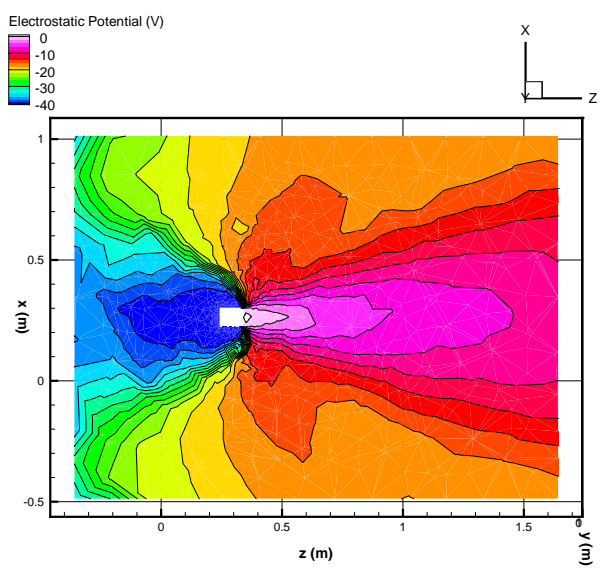

(a) SCN $\Delta t=2.5 \times 10^{-7} \mathrm{~s}$

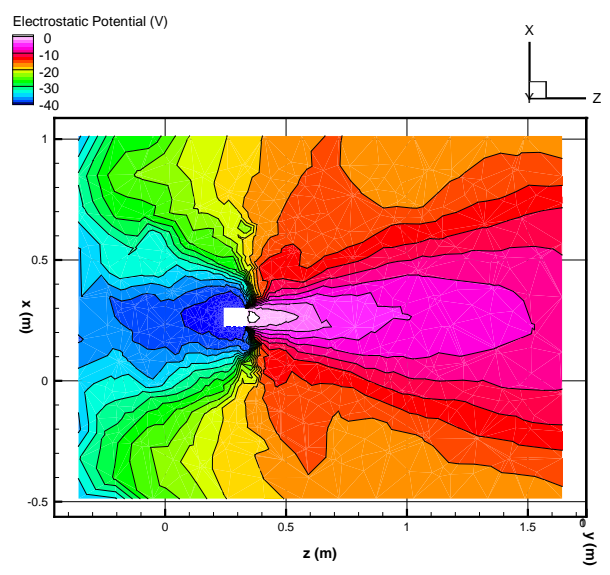

(b) SCN $\Delta t=2.5 \times 10^{-5} \mathrm{~s}$

Figure 13. Final Electrostatic Potential for Coarse Time Step 
time step. The $20 \mathrm{~km} / \mathrm{s}$ neutrals are results of the charge exchange collisions with the high speed ions. The $10 \mathrm{~km} / \mathrm{s}$ neutrals are either from secondary collisions between the high speed neutral and the other particles (such as elastic collisions between ions and neutrals and two neutrals) or from ellastic collisions between the beam ions and the beam neutrals. One point to note is that with the large time step, the high speed neutrals are able to travel entirely through the high density region before any other collision event might occur.
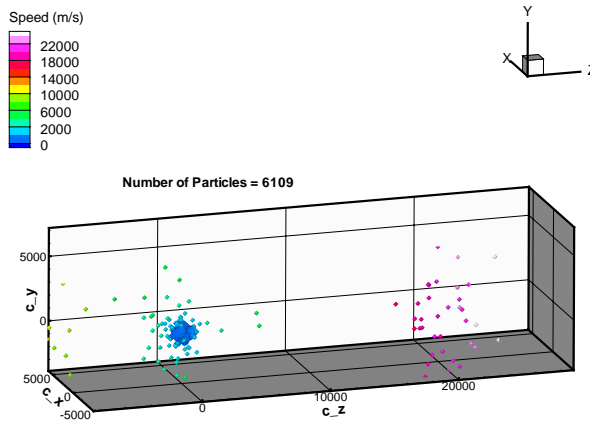

(a) SCN Velocity $\Delta t=2.5 \times 10^{-7} \mathrm{~s}$

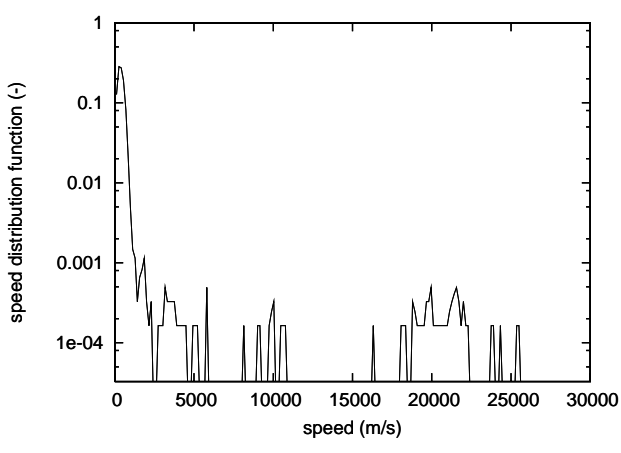

(c) SCN Speed $\Delta t=2.5 \times 10^{-7} \mathrm{~s}$
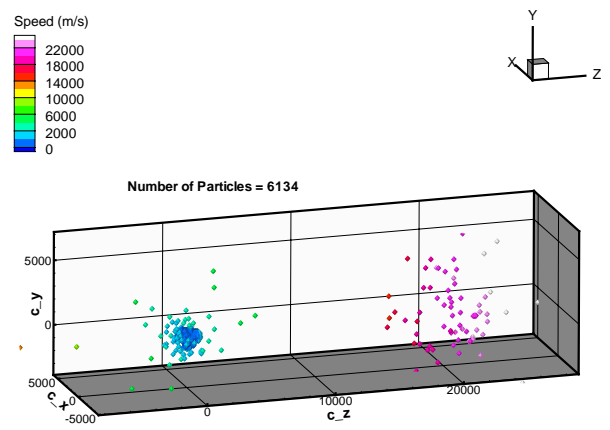

(b) SCN Velocity $\Delta t=2.5 \times 10^{-5} \mathrm{~s}$

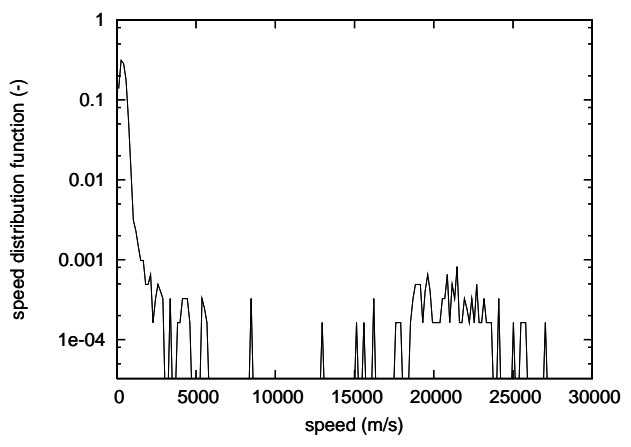

(d) SCN Speed $\Delta t=2.5 \times 10^{-5} \mathrm{~s}$

Figure 14. Neutral Velocity and Speed Distributions $0.1 \mathrm{~m}$ in Front of Thruster for Coarse Time Step

Figure 15 shows the ion sampling within the plume. Again a difference can be seen between the coarse and fine time step cases. First, there is a noticable increase in the number of ions in between the two modes in the distribution. This velocity range, around $10 \mathrm{~km} / \mathrm{s}$, corresponds to the decrease in the distribution of neutrals mentioned above. It appears that with the coarse time step there is not enough of the secondary collisions to decrease the population of the higher energy neutral as well as the moderate energy ions. Notice that these ions are mainly produced by the charge exchange collisions from a previous time step since the plasma source is producing a Maxwellian distribution of ions with the peak of the distribution at $20 \mathrm{~km} / \mathrm{s}$. Thus it appears that the coarse time step is moving the particles out of the high density region too quickly.

The neutral sampling outside the plume is shown in Figure 16 for both the velocity distribution as well as the speed distribution. While the two distributions look similar, there is a more concentrated collection of particles in the $8 \mathrm{~km} / \mathrm{s}$ region for the coarse time step. This is hard to distinguish from the statistical scatter in the data, but this clustering does occur over 8 consecutive velocity bins, so this is likely more than a statistical artifact.

Figure 17 shows the ion sampling outside the plume. In this case there are significant differences between the coarse and fine time step cases. The most notable is that the coarse time step has a bimodal distribution with the second peak rather wide and centered around $10 \mathrm{~km} / \mathrm{s}$. This peak does not appear on the fine time step and must be a result of the coarse time step. Again, the speed is associated with secondary collisions, and if the fast moving particles, which are the only ones that create these particles, travel through the high density region before another collision event is performed, then there would be left over medium speed ions. Also, the width of the main peak, that is center at $4 \mathrm{~km} / \mathrm{s}$ is much larger for the coarse time step than for 

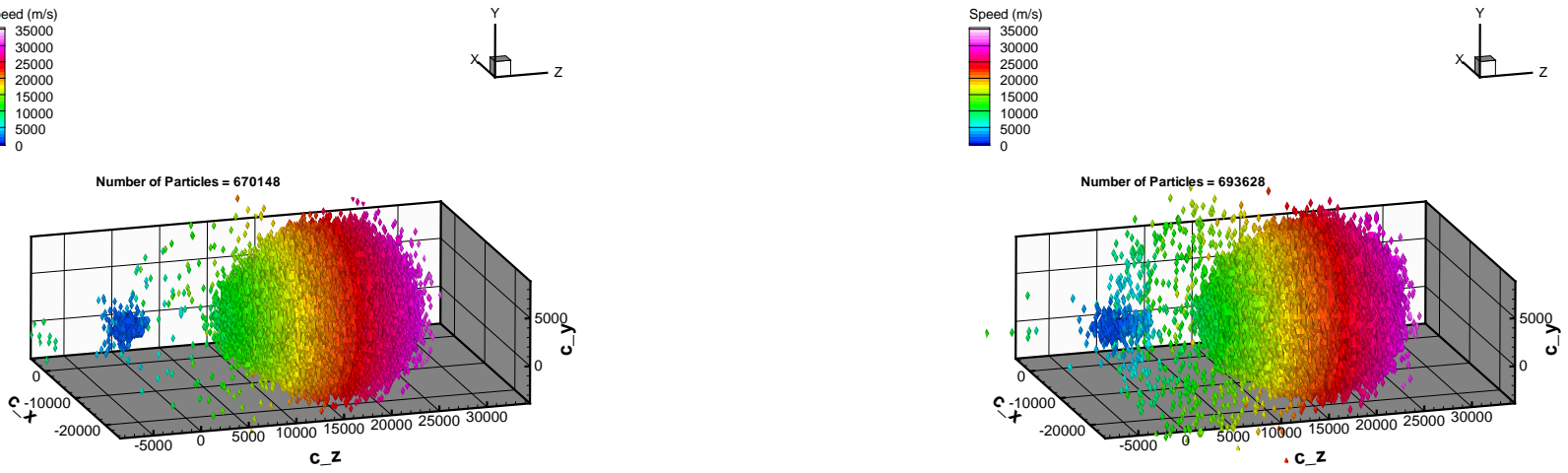

(a) SCN Velocity $\Delta t=2.5 \times 10^{-7} \mathrm{~s}$

(b) SCN Velocity $\Delta t=2.5 \times 10^{-5} \mathrm{~s}$
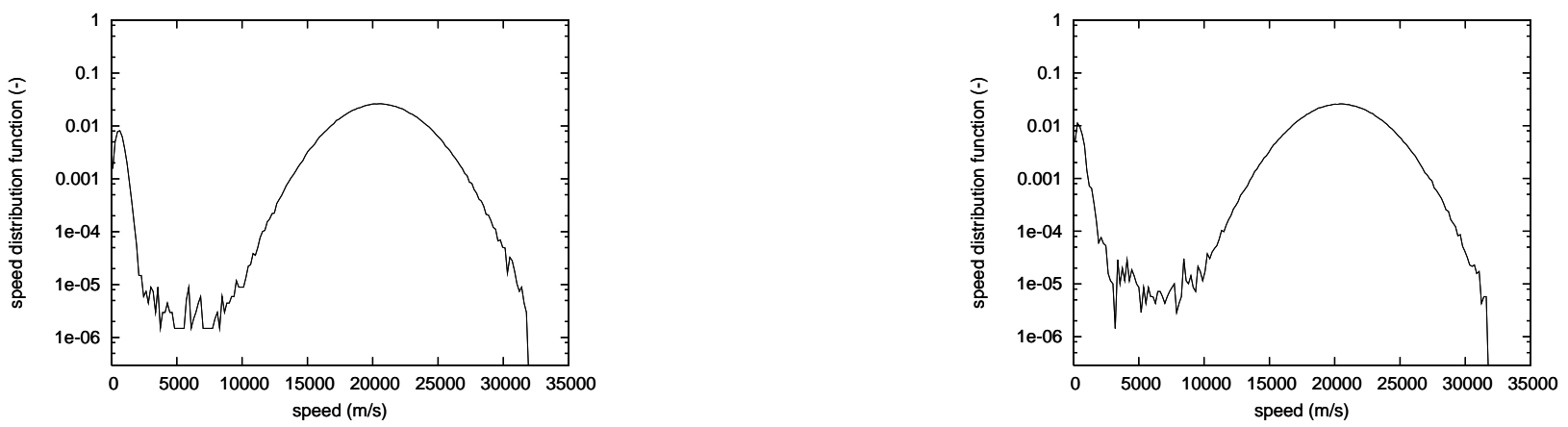

(c) SCN Speed $\Delta t=2.5 \times 10^{-7} \mathrm{~s}$

(d) SCN Speed $\Delta t=2.5 \times 10^{-5} \mathrm{~s}$

Figure 15. Ion Velocity and Speed Distributions $0.1 \mathrm{~m}$ in Front of Thruster for Coarse Time Step 

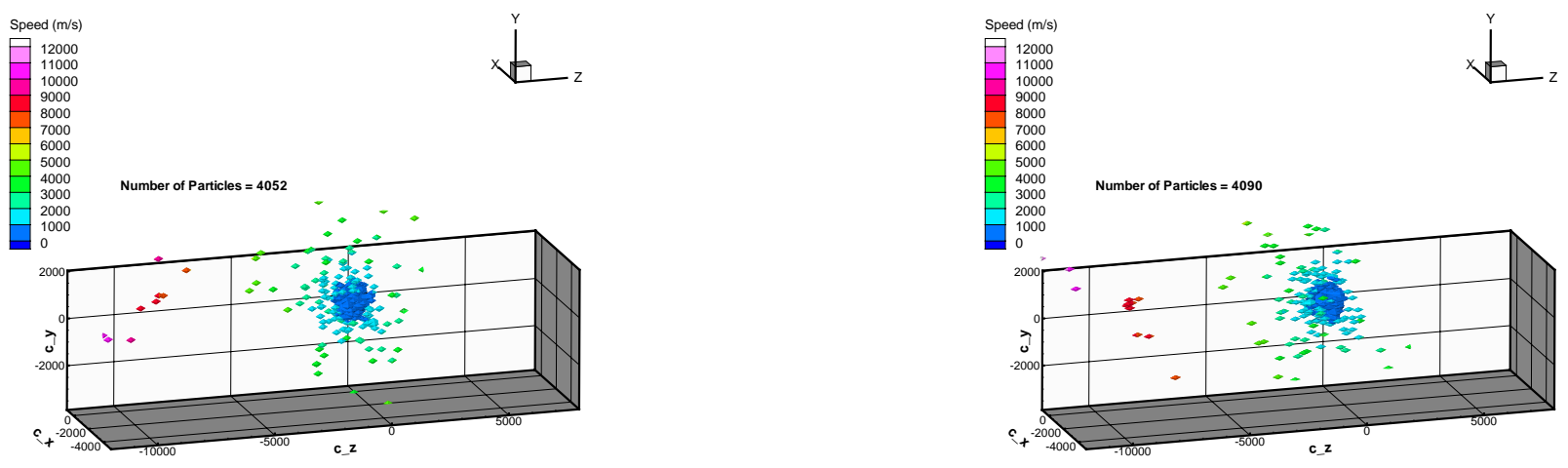

(a) SCN Velocity $\Delta t=2.5 \times 10^{-7} \mathrm{~s}$
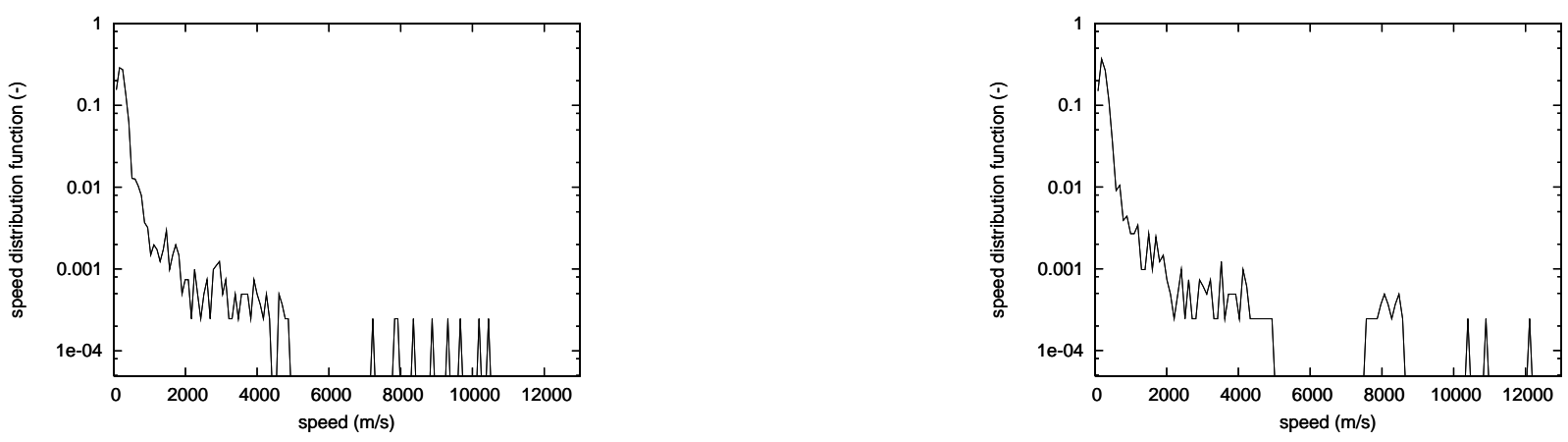

(c) SCN Speed $\Delta t=2.5 \times 10^{-7} \mathrm{~s}$

(d) SCN Speed $\Delta t=2.5 \times 10^{-5} \mathrm{~s}$

Figure 16. Neutral Velocity and Speed Distributions $0.28 \mathrm{~m}$ Above Thruster Face for Coarse Time Step 
the fine time step. There is also a corresponding increase in the speed distribution function value at the peak. Again since this peak is most likely a result of multiple collisions occuring before the particle leaves the high density region, the coarser time step can again account for this difference.

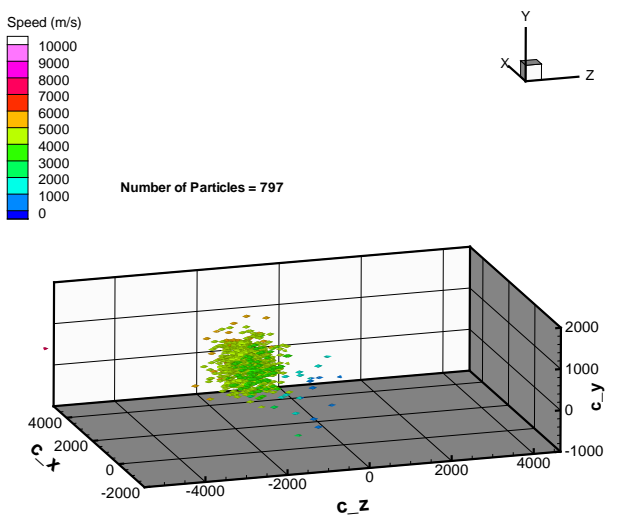

(a) SCN Velocity $\Delta t=2.5 \times 10^{-7} \mathrm{~s}$

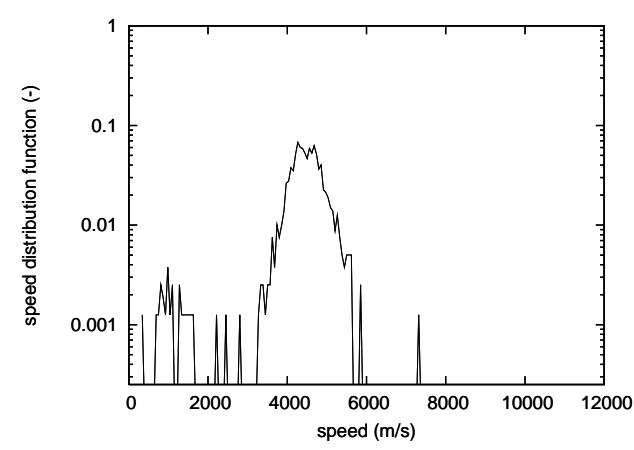

(c) SCN Speed $\Delta t=2.5 \times 10^{-7} \mathrm{~s}$

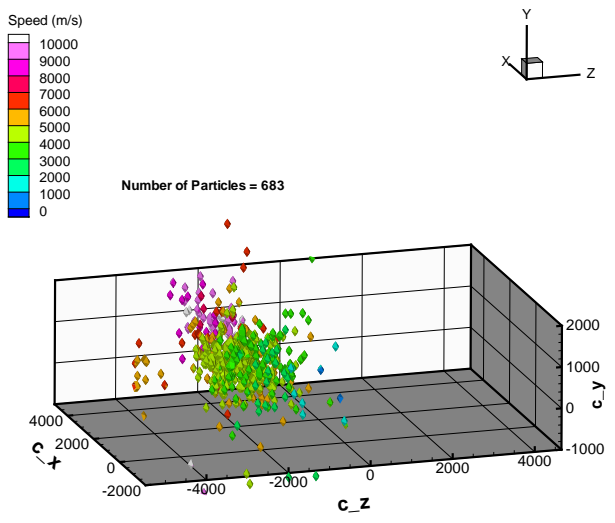

(b) SCN Velocity $\Delta t=2.5 \times 10^{-5} \mathrm{~s}$

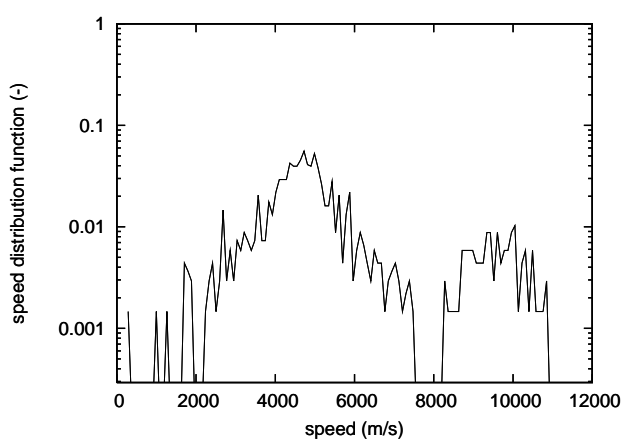

(d) SCN Speed $\Delta t=2.5 \times 10^{-5} \mathrm{~s}$

Figure 17. Ion Velocity and Speed Distributions $0.28 \mathrm{~m}$ Above Thruster Face for Coarse Time Step

It appears that there is a significant coupling between the particle time step and the ability to capture all of the secondary collisions that are occurring. Even though the time step was fine enough for the collision modeling characteristic time step, it appears that the rapid particle density variation in front of the plasma source is causing the collision characteristic time step calculation to be too large. Also, notice that while some of these effects are noticeable in the gross perspective of the flow field, the significant differences are only apparent with the observation of the velocity distribution functions.

\section{Subcycling Solution}

Now that is apparent the the coarse time step does not capture a number of significant flow features, an analysis of the improvements associated with the subcycling algorithm can be performed. The subcycling algorithm uses the coarse time step, $2.5 \times 10^{-5} \mathrm{~s}$, for the slow particle time step (i.e., slow neutrals) and the fine time step, $2.5 \times 10^{-7} \mathrm{~s}$, for the fast particle time step (i.e., for the fast ions and neutrals). Table 4 shows the resulting collision rates along with the fine and coarse time step collision rates for comparison.

The first thing to notice from this figure is that the subcycling scheme results in a decrease of computational time from $58.9 \mathrm{hr}$ to $13.7 \mathrm{hr}$ compared to the fine time step case. Unfortunately, the collision rates are nearly identical to the coarse time step rates and are significantly lower than the fine time step results (with the same exception of the $\mathrm{Xe}-\mathrm{Xe}$ elastic collision rate).

While significant differences are seen in the collision rates, Figure 18 shows the evolution of the total number of neutrals and ions as well as the total number of particles is very similar. Thus, the differences between the subcycling case and the fine time step case again must be for only a small, but significant, fraction of the total particles. 
Table 4. Collision Rates for Subcycling Case

\begin{tabular}{ccccccc} 
& $\begin{array}{c}\text { Compute } \\
\text { Scheme }\end{array}$ & $\begin{array}{c}\text { Time } \\
\text { Time }[\mathrm{hr}]\end{array}$ & $\begin{array}{c}\text { Total } \\
\text { Step [s] }\end{array}$ & $\begin{array}{c}\text { Xe-Xe+ Charge } \\
\text { Collisions [\#/s] }\end{array}$ & $\begin{array}{c}\text { Xe-Xe } \\
\text { Exchange [\#/s] }\end{array}$ & $\begin{array}{c}\text { Xe-Xe }{ }^{+} \\
\text {Elastic [\#/s] }\end{array}$ \\
\hline Elastic [\#/s] & 58.9 & $2.5 \times 10^{-7}$ & $5.325 \times 10^{7}$ & $5.069 \times 10^{7}$ & $2.560 \times 10^{5}$ & $2.303 \times 10^{6}$ \\
SCN & 1.73 & $2.5 \times 10^{-5}$ & $4.945 \times 10^{7}$ & $4.726 \times 10^{7}$ & $2.572 \times 10^{5}$ & $1.936 \times 10^{6}$ \\
SCY & 17.3 & $2.5 \times 10^{-5}$ & $4.966 \times 10^{7}$ & $4.745 \times 10^{7}$ & $2.535 \times 10^{5}$ & $1.963 \times 10^{6}$
\end{tabular}

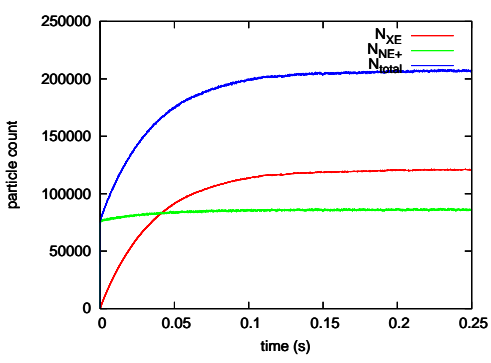

(a) $\mathrm{SCN} \Delta t=2.5 \times 10^{-7} \mathrm{~s}$

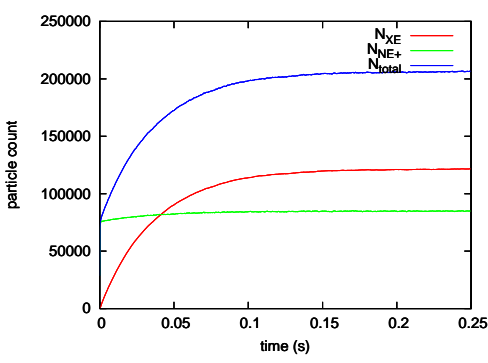

(b) SCN $\Delta t=2.5 \times 10^{-5} \mathrm{~s}$

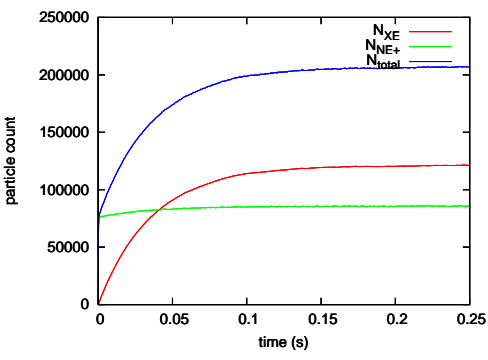

(c) SCY $\Delta t=2.5 \times 10^{-5} \mathrm{~s}$

Figure 18. Global Particle Counts for Subcycling Case

Figure 19 shows the final number density for the neutrals for the subcycling case as well as the fine and coarse time step cases. This case again shows that there is little difference between the neutral number densities for the subcycling case. Thus, the overall neutral distribution is fairly insensitive to the computational time step. However, as was the case for the coarse time step, it is expected that there will be differences that are not observable in this perspective.

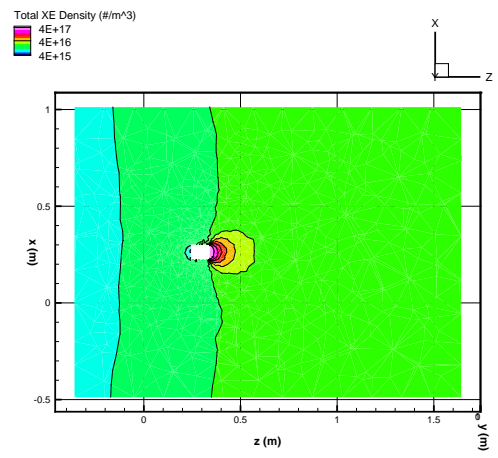

(a) $\operatorname{SCN} \Delta t=2.5 \times 10^{-7} \mathrm{~s}$

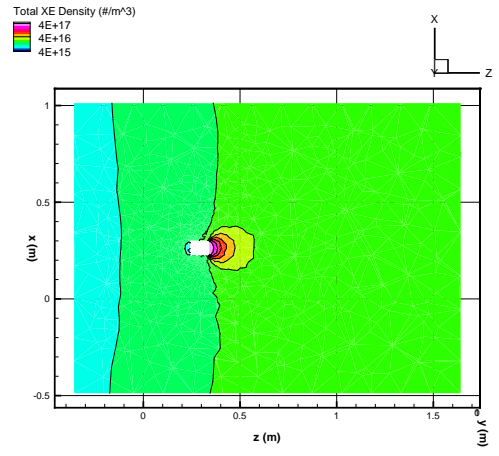

(b) $\mathrm{SCN} \Delta t=2.5 \times 10^{-5} \mathrm{~s}$

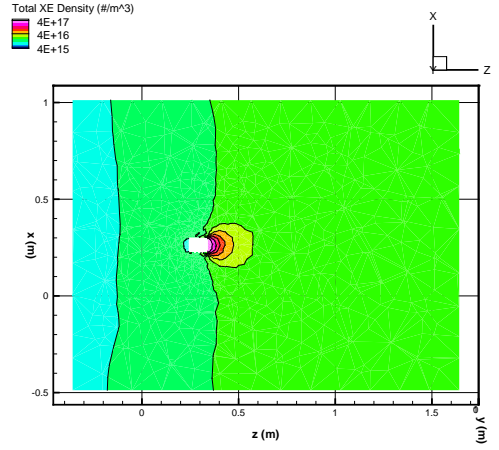

(c) SCY $\Delta t=2.5 \times 10^{-5} \mathrm{~s}$

Figure 19. Final Neutral Number Density for Subcycling Case

Figure 20 shows the final number density for the ions for the subcycling case as well as the fine and coarse time step cases. Unlike the coarse time step case, the ion number density is very close to the fine time step case. The outer wings of the plume are captured, and the ion neutralization at the back of the plasma source is also captured. Therefore, the subcycling is drastically improving the capabilities of capturing the ion spatial distribution.

Figure 21 shows the final electrostatic potential for the subcycling case as well as the fine and coarse time step cases. This again shows that the subcycling case and the fine time step cases are quite similar. This is to be expected since the electrostatic potential is directly related to the ion distribution. Even the potential drop behind the thruster seen in the fine time step case is captured in the subcycling case.

While the collision rates are different, the subcycling case has so far improved the ion number density distribution compared to the coarse time step case and has shown no difference in the neutral number density distribution. 


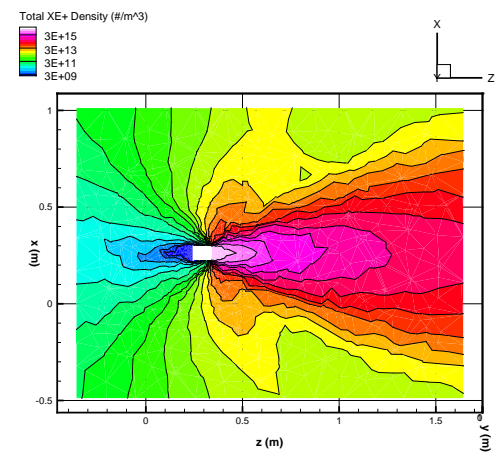

(a) $\operatorname{SCN} \Delta t=2.5 \times 10^{-7} \mathrm{~s}$

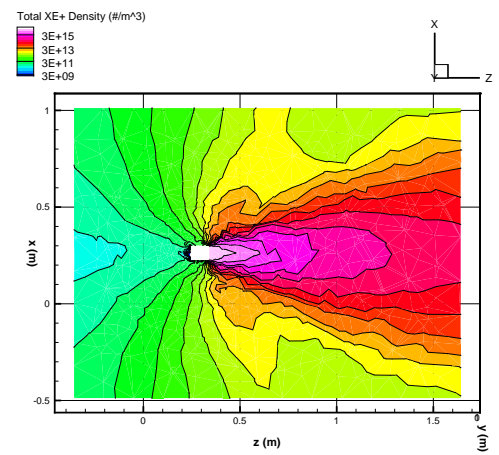

(b) $\mathrm{SCN} \Delta t=2.5 \times 10^{-5} \mathrm{~s}$

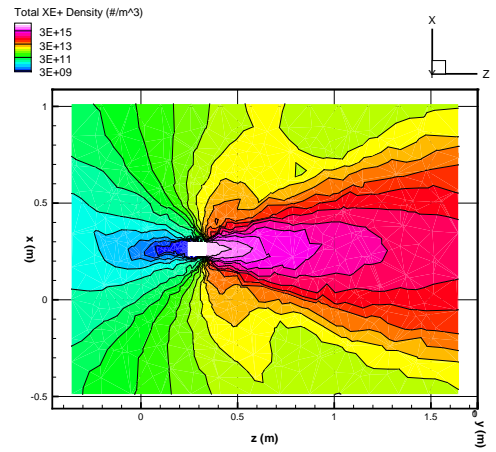

(c) SCY $\Delta t=2.5 \times 10^{-5} \mathrm{~s}$

Figure 20. Final Ion Number Density for Subcycling Case

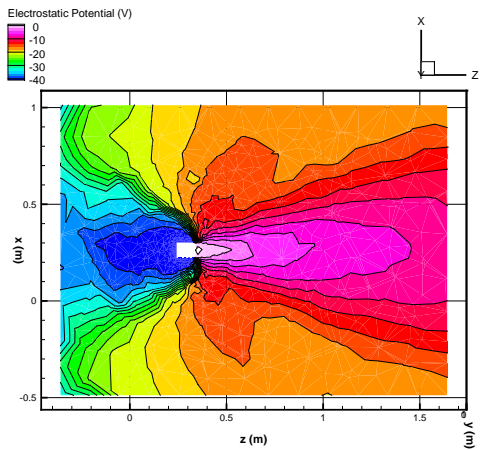

(a) $\operatorname{SCN} \Delta t=2.5 \times 10^{-7} \mathrm{~s}$

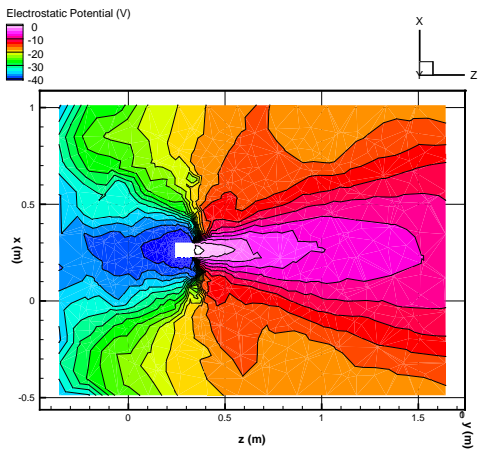

(b) $\operatorname{SCN} \Delta t=2.5 \times 10^{-5} \mathrm{~s}$

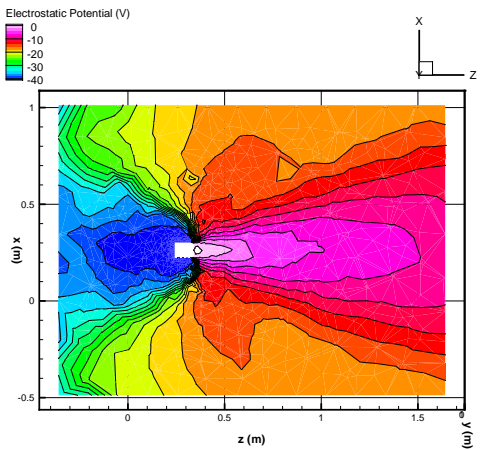

(c) SCY $\Delta t=2.5 \times 10^{-5} \mathrm{~s}$

Figure 21. Final Electrostatic Potential for Subcycling Case 
Next, the local properties of the plasma will be compared between the subcycling case and the fine and coarse solution. The neutral sampling within the plume is shown in Figure 22 for both the velocity distribution as well as the speed distribution. Unfortunately, the subcycling cases looks much more similar to the coarse time step case and has significant differences with the fine time step case. The same arguments about the coarse time step differences also seem to apply here. While the neutrals are propagating at the fine time step, there is still no mechanism to get these neutrals to participate in collision events while they reside in the high density regions since collisions are only computed at the coarse time scale.

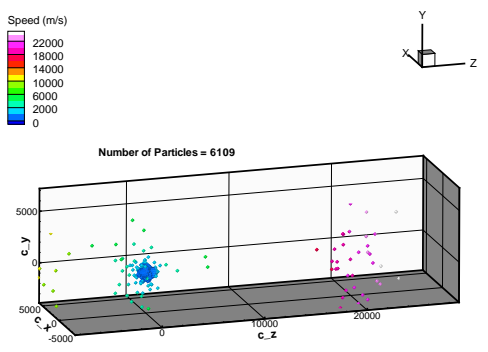

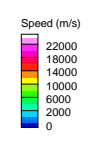

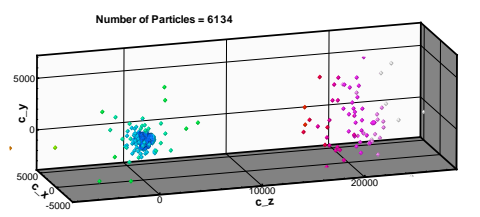

(b) SCN Velocity $\Delta t=2.5 \times 10^{-5} \mathrm{~s}$

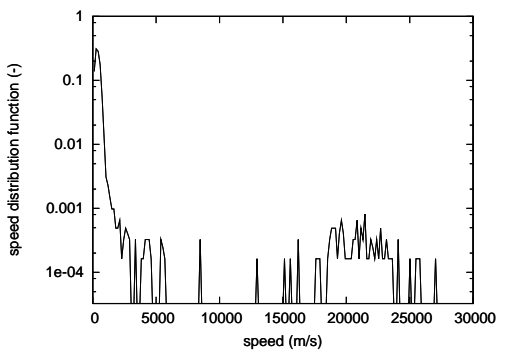

(e) SCN Speed $\Delta t=2.5 \times 10^{-5} \mathrm{~s}$

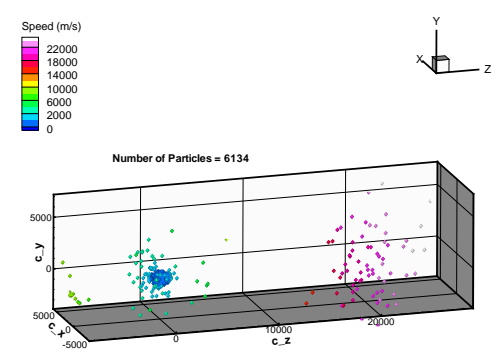

(c) SCY Velocity $\Delta t=2.5 \times 10^{-5} \mathrm{~s}$

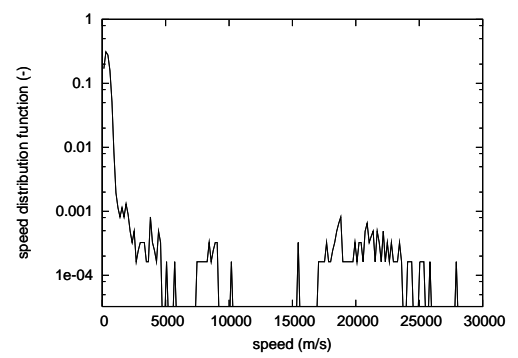

(f) SCY Speed $\Delta t=2.5 \times 10^{-5} \mathrm{~s}$

(d) SCN Speed $\Delta t=2.5 \times 10^{-7} \mathrm{~s}$

Figure 22. Neutral Velocity and Speed Distributions $0.1 \mathrm{~m}$ in Front of Thruster for Subcycling Case

Figure 23 shows the ion sampling within the plume. This subcycling case also shows significant differences between the subcycling and fine time steps and is very similar to the coarse time step. It appears that this, too might be attributed to the secondary collisions discussed previously.

The neutral sampling outside the plume is shown in Figure 24 for both the velocity distribution as well as the speed distribution. While it is not certain that the $8 \mathrm{~km} / \mathrm{s}$ region in the coarse time step is caused by statistical scatter, it is worth noting that the subcycling case does not demonstrate this feature. Otherwise, the subcycling case looks very similar to the fine time step case.

Figure 25 shows the ion sampling outside the plume. This case shows drastic improvements from the coarse time step. The subcycling case does not have the secondary peak in the $10 \mathrm{~km} / \mathrm{s}$ range and has a similarly narrow speed range around the most probable speed. It is apparent that the subcycling does significantly improve the particle modeling outside the high density plume region. This is most likely due to the fact that the trajectory of the high speed ions is significantly improved with the fine time step, and thus these high speed ions are more effected by the electrostatic potential field.

\section{Conclusions}

The subcycling acceleration scheme within the AQUILA plasma modeling module of COLISEUM was investigated to determine how effective it is in capturing the local plasma properties. First, the simulation was demonstrated to be capable of converging to a solution for a sufficiently fine time step. This solution was the used to compare the performance of the simulation at a much coarser time step. This showed several deficiencies in the coarse time step solution. These were mainly focused on the fact that the high speed particles are leaving the high density region where multiple collisions are expected to occur after one or two 


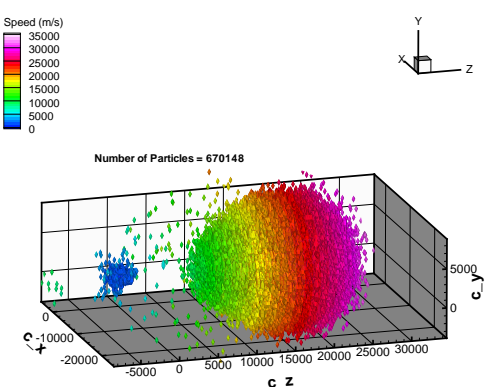

(a) SCN Velocity $\Delta t=2.5 \times 10^{-7} \mathrm{~s}$

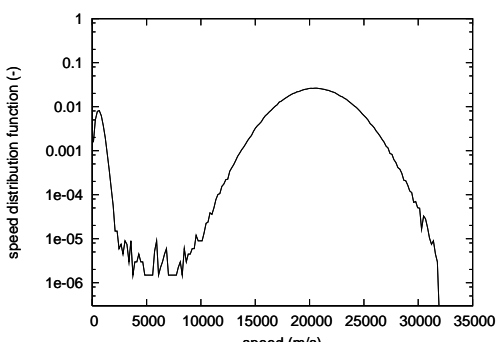

(d) SCN Speed $\Delta t=2.5 \times 10^{-7} \mathrm{~s}$

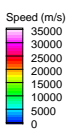

in

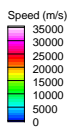

Y
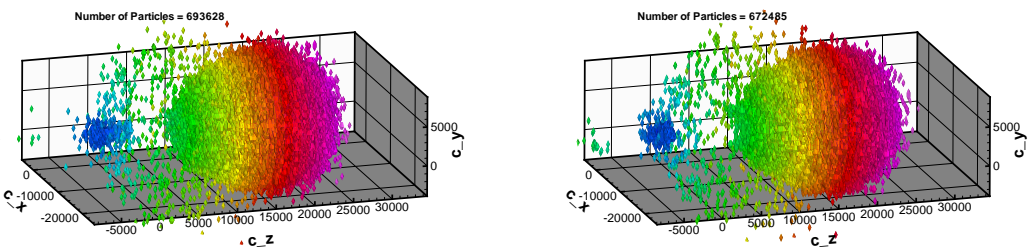

(b) SCN Velocity $\Delta t=2.5 \times 10^{-5} \mathrm{~s}$

(c) SCY Velocity $\Delta t=2.5 \times 10^{-5} \mathrm{~s}$

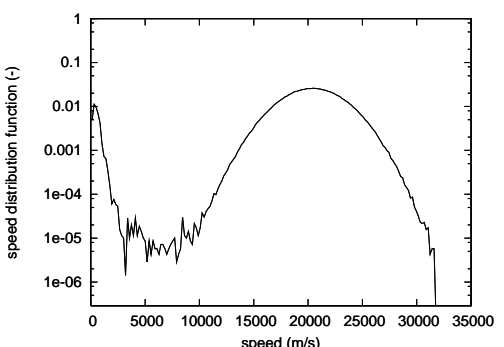

(e) SCN Speed $\Delta t=2.5 \times 10^{-5} \mathrm{~s}$

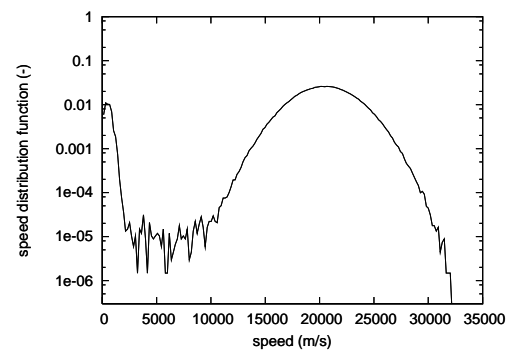

(f) SCY Speed $\Delta t=2.5 \times 10^{-5} \mathrm{~s}$

Figure 23. Ion Velocity and Speed Distributions $0.1 \mathrm{~m}$ in Front of Thruster for Subcycling Case
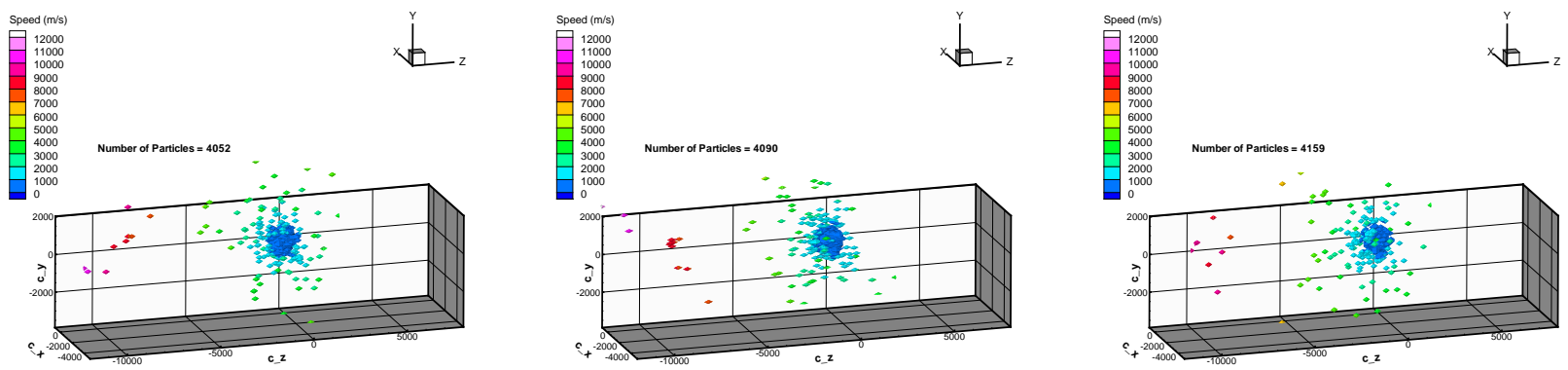

(a) SCN Velocity $\Delta t=2.5 \times 10^{-7} \mathrm{~s}$

(b) SCN Velocity $\Delta t=2.5 \times 10^{-5} \mathrm{~s}$

(c) SCY Velocity $\Delta t=2.5 \times 10^{-5} \mathrm{~s}$
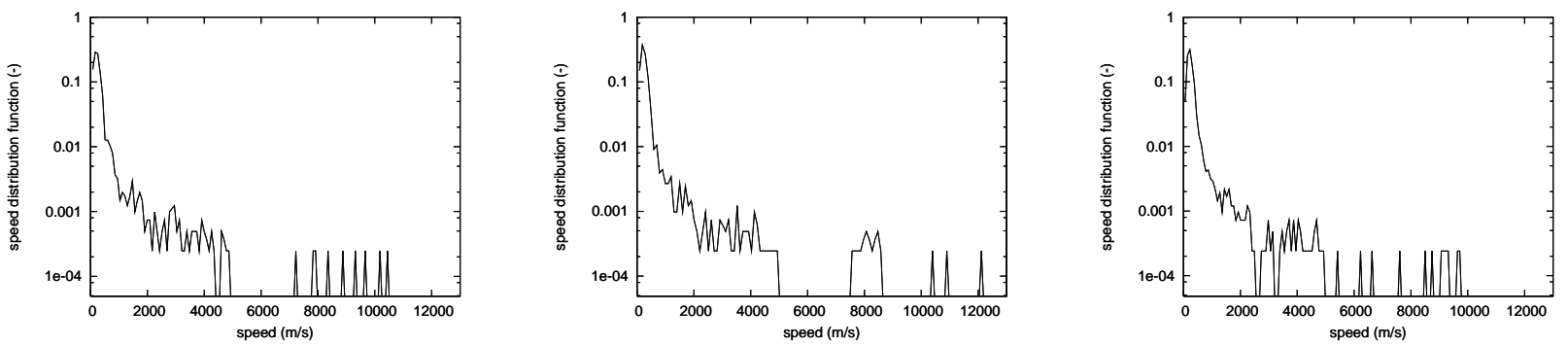

(d) SCN Speed $\Delta t=2.5 \times 10^{-7} \mathrm{~s}$

(f) SCY Speed $\Delta t=2.5 \times 10^{-5} \mathrm{~s}$

Figure 24. Neutral Velocity and Speed Distributions $0.28 \mathrm{~m}$ Above Thruster Face for Subcycling Case 


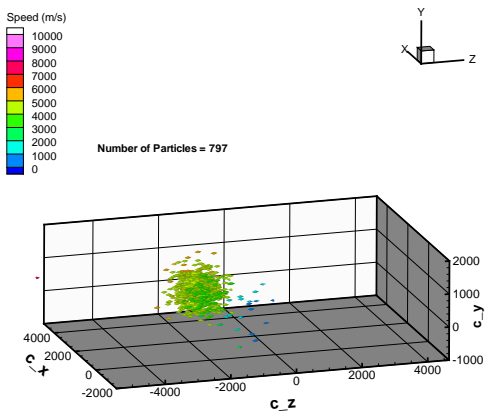

(a) SCN Velocity $\Delta t=2.5 \times 10^{-7} \mathrm{~s}$

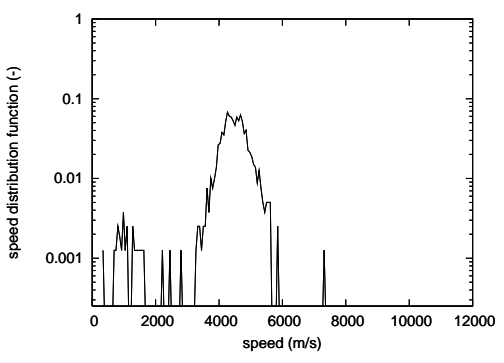

(d) SCN Speed $\Delta t=2.5 \times 10^{-7} \mathrm{~s}$

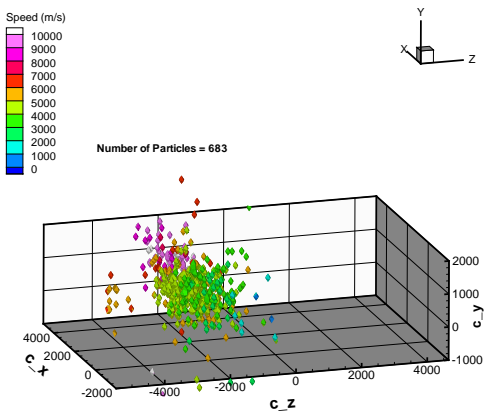

(b) SCN Velocity $\Delta t=2.5 \times 10^{-5} \mathrm{~s}$

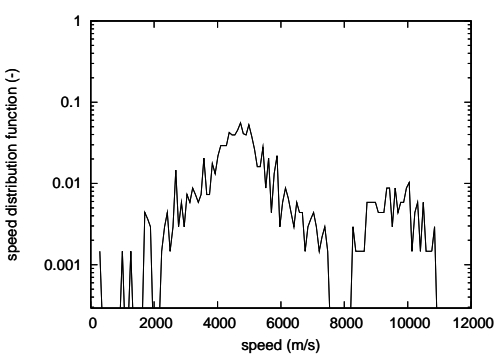

(e) SCN Speed $\Delta t=2.5 \times 10^{-5} \mathrm{~s}$

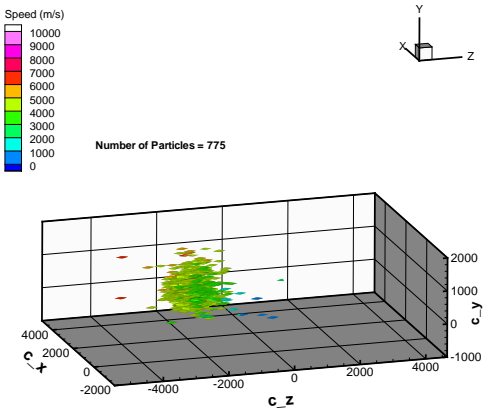

(c) SCY Velocity $\Delta t=2.5 \times 10^{-5} \mathrm{~s}$

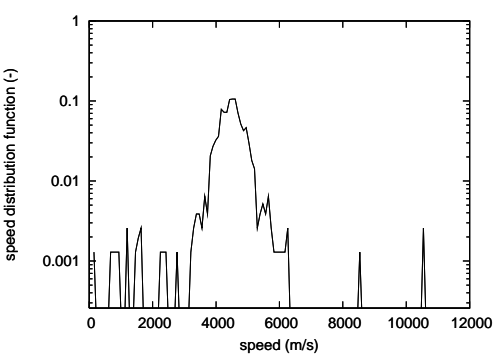

(f) SCY Speed $\Delta t=2.5 \times 10^{-5} \mathrm{~s}$

Figure 25. Ion Velocity and Speed Distributions $0.28 \mathrm{~m}$ Above Thruster Face for Subcycling Case

time steps. In addition the coarse time step resulted in the ion trajectories being significantly off which resulted in large differences in the ion density distribution compared to the fine time step case.

The subcycling case improved the modeling of the number densities of the ions and neutrals compared to the coarse time step simulation with a compute time speedup of a factor of 3.4. It also significantly improved the modeling of the lower density region outside the main plume. In this region the velocity distribution functions for the neutrals and ions were both very similar to the fine time step case. This shows that improving the modeling of the electrostatic forces, via finer time steps to propagate the ions and the force calculations, does improve the modeling of the plasma. For the higher density region of the main beam of the plasma, the subcycling showed no improvement compared to the coarse time step solution. While this appears to be rooted in the fact that a high speed particle can travel through the entire high density plume region in one time step and thus not participate in the additional collisions that appear to produce the different features of the fine time step solution. An alternative explanation to this behavior could be that the collision model is not providing consistent results at the different time scales. Suppose the collision model was selecting too many collisions to occur at the finer time step because the selection criteria was slightly off. This would result in a non-physical increase in additional collisions that the high speed particles would be participating in. This would mean that the fine time step solution was not correct.

It is apparent from this study that the collision model used with AQUILA needs further validation. Although AQUILA has been successful in previous studies that examined the aggregate properties, ${ }^{2,3,10,12}$ a more detailed examination of the collision algorithm is needed. The combination of using this algorithm in which variable species weighting is used with the subcycling technique, i.e. separate time steps for high speed and low speed particles, requires more attention. For this type of rarefield flow in which collisions result in orders of magnitude changes in velocity, it may be necessary to use a different collision algorithm and/or conserve momentum and energy for each collision. A future study of the effects of various collision algorithms on the local velocity distribution function should be performed to assess their ability to handle the variable species weighting that is used within AQUILA. 


\section{References}

${ }^{1}$ Fife, J. M., Gibbons, M. R., Hargus, W. A., VanGilder, D. B., and Kirtley, D. E., "3-D Computation of Surface Sputtering and Redeposition Due to Hall Thruster Plumes," 28 $8^{\text {th }}$ International Electric Propulsion Conference, March 17-21 2003.

${ }^{2}$ Santi, M., Cheng, S., Celik, M., Martinez-Sanchez, M., and Peraire, J., "Futher Development and Preliminary Results of the Aquila Hall Thruster Plume Model," 39 ${ }^{\text {th }}$ AIAA/ASEE/SAE/ASEE Joint Propulsion Conference, No. AIAA-2003-4873, Huntsville, AL, July 20-23 2003.

${ }^{3}$ Gibbons, M. R., Kirtley, D. E., VanGilder, D. B., and Fife, J. M., "Flexible Three-Dimensional Modeling of Electric Thrusters in Vacuum Chambers," $39^{\text {th }}$ AIAA/ASEE/SAE/ASEE Joint Propulsion Conference, No. AIAA-2003-4872, Huntsville, AL, July 20-23 2003.

${ }^{4}$ Gibbons, M. R., Santi, M., VanGilder, D. B., and Fife, J. M., "Simulation of Plasma Expansion Using a Two-Timescale Accelerated Particle-in-Cell Method," $42^{\text {nd }}$ AIAA Aerospace Sciences Meeting and Exhibit, No. AIAA-2004-0154, Reno, NV, January 5-8 2004 .

${ }^{5}$ Roussel, J.-F., Bernard, J., and Garnier, Y., "Numerical Simulation of Induced Environment, Sputtering and Contamination of a Satellite Due to Electric Propulsion," $2^{\text {nd }}$ European Spacecraft Propulsion Conference, Noordwijk, the Netherlands, May 27-29 1997.

${ }^{6}$ Gardner, B., Davis, V. A., Katz, I., and J., M. M., "Hall Current Thruster Plume Modeling - A Diagnostic Tool for Spacecraft Subsystem Impact," $39^{\text {th }}$ Aerospace Sciences Meeting, No. AIAA-2001-0964, Reno, NV, January 8-11 2001.

${ }^{7}$ Nannenberg, K., Khayms, V., Emgushov, B., and Werthman, L., "Validation of Hall Thruster Plume Sputter Model," $3^{\text {th }}$ AIAA/ASME/SAE/ASEE Joint Propulsion Conference, No. AIAA-2001-3986, Salt Lake City, UT, July 8-11 2001.

${ }^{8}$ Yamamura, Y., Itikawa, Y., and Itoh, N., "Angular Dependence of Sputtering Yields of Monoatomic Solids," Tech. Rep. IPPJ-AM-26, Nagoya, Japan, 1983.

${ }^{9}$ Brieda, L., Development of the DRACO ES-PIC code and Fully-Kinetic Simulation of Ion Beam Neutralization, Master's thesis, Virginia Polytechnic Institute and State University, Blacksburg, VA, 2005.

${ }^{10}$ Santi, M., Hall Thruster Plume Simulation Using a hybrid-PIC Algorithm, Master's thesis, Massachusetts Institute of Technology, Cambridge, MA, 2003.

${ }^{11}$ Birdsall, C. K. and Langdon, A. B., Plasma Physics via Computer Simulation, McGraw-Hill, New York, 1985.

${ }^{12}$ Celik, M., Santi, M., Cheng, S., Martinez-Sanchez, M., and Peraire, J., "Hybrid-PIC Simulation of a Hall Thruster Plume on an Unstructured Grid with DSMC Collisions," 28 $8^{\text {th }}$ International Electric Propulsion Conference, March 17-21 2003.

${ }^{13}$ Bird, G. A., Molecular Gas Dynamics and the Direct Simulation of Gas Flows, Oxford University Press, Oxford, 1994.

${ }^{14} \mathrm{Oh}$, D. Y. and Hastings, D. E., "Experimental Verification of a PIC-DSMC Model for Hall Thruster Plumes," 32 ${ }^{\text {nd }}$ AIAA/ASME/SAE/ASEE Joint Propulsion Conference, No. AIAA-1996-3196, Lake Buena Vista, FL, July 1-3 1996.

${ }^{15}$ Pullins, S., Chiu, Y.-H., Levandier, D. J., and Dressler, R. A., "Ion Dynamics in Hall Effect and Ion Thrusters - Xe ${ }^{+}$ + Xe Symmetric Charge Transfer," $38^{t h}$ Aerospace Sciences Meeting and Exhibit, No. AIAA-2000-0603, Reno, NV, January 10-132000.

${ }^{16}$ U.S. Air Force Research Laboratory, Rocket Propulsion Division, Spacecraft Branch, Edwards AFB, CA, COLISEUM User's Manual, 2005. 